UFIFT-QG-20-02

CP3-20-10

\title{
Single Graviton Loop Contribution to the Self-Mass of a Massless, Conformally Coupled Scalar on de Sitter Background
}

\author{
D. Glavan ${ }^{1 *}$, S. P. Miao ${ }^{2 \star}$, T. Prokopec ${ }^{3 \dagger}$ and R. P. Woodard ${ }^{4 \ddagger}$ \\ ${ }^{1}$ Centre for Cosmology, Particle Physics and Phenomenology (CP3) \\ Université catholique de Louvain, Chemin du Cyclotron 2, 1348 \\ Louvain-la-Neuve, BELGIUM \\ ${ }^{2}$ Department of Physics, National Cheng Kung University \\ No. 1 University Road, Tainan City 70101, TAIWAN

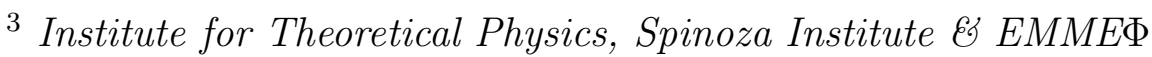 \\ Utrecht University, Postbus 80.195, 3508 TD Utrecht, THE \\ NETHERLANDS \\ ${ }^{4}$ Department of Physics, University of Florida, \\ Gainesville, FL 32611, UNITED STATES
}

\begin{abstract}
We use a simplified formalism to re-compute the single graviton loop contribution to the self-mass of a massless, conformally coupled scalar on de Sitter background which was originally made by Boran, Kahya and Park [1]3. Our result resolves the problem with the flat space correspondence limit that was pointed out by Fröb [4]. We discuss how this computation will be used in a long-term project to purge the linearized effective field equation of gauge dependence.
\end{abstract}

PACS numbers: 04.50.Kd, 95.35.+d, 98.62.-g

* e-mail: drazen.glavan@uclouvain.be

* e-mail: spmiao5@mail.ncku.edu.tw

† e-mail: T.Prokopec@uu.nl

‡ e-mail: woodard@phys.ufl.edu 


\section{Introduction}

Computing fully regulated and renormalized quantum gravitational loop corrections is not easy even on flat space background, and is especially challenging on curved backgrounds. However, the discovery of a relatively simple gauge [5,6] for de Sitter background has facilitated computations of the graviton self-energy [7], the self-energy of massless [8] and massive [9] fermions, the self-mass of a massless, minimally coupled [10] and conformally coupled [1,2] scalars and the vacuum polarization [11. These 1PI (one-particle irreducible) 2-point functions can be used to quantum-correct the linearized effective field equations to infer loop corrections to forces and mode functions using the Schwinger-Keldysh formalism [12]. For example, the vacuum polarization $\left.i^{[\mu} \Pi^{\nu}\right]\left(x ; x^{\prime}\right)$ changes Maxwell's equation in background metric $g_{\mu \nu}$ to [13, 14],

$$
\partial_{\nu}\left[\sqrt{-g} g^{\nu \rho} g^{\mu \sigma} F_{\rho \sigma}(x)\right]+\int d^{4} x^{\prime}\left[{ }^{\mu} \Pi^{\nu}\right]\left(x ; x^{\prime}\right) A_{\nu}\left(x^{\prime}\right)=J^{\mu}(x),
$$

where $J^{\mu}(x)$ is the current density, $A_{\mu}$ is the vector potential, and $F_{\mu \nu} \equiv$ $\partial_{\mu} A_{\nu}-\partial_{\nu} A_{\mu}$ is the field strength. Linearized effective field equations have been solved for one loop corrections to the graviton mode function (in Hartree approximation) [15], to the massless fermion mode function [16], to the mode functions of minimally coupled [17] and conformally coupled [3] scalars, to the photon mode function [18], and to electromagnetic forces [19].

The results of these studies are fascinating because they often show large logarithmic corrections to mode functions and exchange potentials. So although the inflationary loop counting parameter of $G H^{2} \sim 10^{-10}$ is small, the large logarithms can make quantum corrections become arbitrarily large at late times and long distances. However, a note of caution arises from the observation that any graviton loop correction is liable to depend upon the gauge fixing procedure. This can be seen explicitly in the ability to make flat space corrections vary from plus infinity to minus infinity [20]. Although the flat space gauge dependence must persist in de Sitter results which survive taking the Hubble parameter to zero, they might have been absent from the uniquely de Sitter effects responsible for the large logarithms [21. However, the one time this was checked by making the vastly more difficult computation of the vacuum polarization in a different gauge [22], it was found that the the coefficient of the large logarithmic correction to the photon field strength does change [23]. 
A way forward is provided by the recent insight that gauge dependence in the effective field equations arises from having ignored quantum gravitational corrections from the source which excites the effective field and from the observer who measures it [24]. It is possible to interpret these source and observer effects as corrections to the 1PI 2-point function using a series of relations derived by Donoghue [25, 26]. When this is done on flat space all dependence on the 2-parameter family of Poincaré invariant gauges drops out of the linearized effective field equation for a minimally coupled scalar [24], and the equation is presumably completely independent of the gauge fixing procedure. We seek to include source and observer corrections to 1PI 2point functions computed on de Sitter background. The tensor analysis can be simplified by working with a scalar, however, the minimally coupled case is undesirable for two reasons. First, as one can see from the electromagnetic analog (11), one loop corrections are sourced by the integral of classical solutions against the 1PI 2-point function, and neither the mode function [27] nor the exchange potential [28] is especially simple for the massless, minimally coupled scalar. Second, it is already known that there are no logarithmic enhancements to the massless, minimally coupled scalar mode function because gravity only couples to the rapidly redshifting kinetic energy [17].

Getting significant corrections seems to require gravitational couplings such as spin that do not redshift [29]. For scalars that implies either a conformal coupling or a mass. The many simplifications associated with the massless, conformally coupled scalar support selecting this system for the first analysis of source and observer corrections on de Sitter background. The uncorrected 1PI 2-point function has been computed by Boran, Kahya and Park [1,2], but their result was complicated by the decision to express it using de Sitter covariant inverse differential operators 1 Fröb has also reported a problem with the flat space limit [4]. For these reasons we will here carry out an independent computation, taking full advantage of the scalar's conformal invariance to derive a considerably simpler result with the correct flat space correspondence limit. Section 2 presents the relevant Feynman rules. The primitive one graviton contribution is computed in section 3, and its renormalization is accomplished in section 4 . Section 5 discusses the role of this result in our program of deriving gauge-independent corrections to the scalar mode function and exchange potential.

\footnotetext{
${ }^{1}$ This decision was made out of deference to concerns over the breaking of de Sitter invariance by the graviton propagator which no longer seem to be an issue [30 39.
} 


\section{Feynman Rules}

The purpose of this section is to present the Feynman rules necessary for our calculation. We first give the action, both in terms of the original and conformally rescaled fields. Then we express the self-mass as a free expectation value of first and second variations of the action. The various interactions are somewhat simplified by using the trace-reversed graviton field. We close by reviewing the scalar and graviton propagators.

The bare Lagrangian for gravity ( $D$-dimensional spacelike metric $g_{\mu \nu}$ ) plus a massless, conformally coupled scalar $\phi$ is,

$$
\mathcal{L}=\frac{\left[R-(D-2)(D-1) H^{2}\right] \sqrt{-g}}{16 \pi G}-\frac{1}{2} \partial_{\mu} \phi \partial_{\nu} \phi g^{\mu \nu} \sqrt{-g}-\frac{1}{8}\left(\frac{D-2}{D-1}\right) \phi^{2} R \sqrt{-g},
$$

where $G$ is Newton's constant and $H$ is the Hubble constant. The background geometry is,

$$
d s^{2}=a^{2}\left[-d \eta^{2}+d \vec{x} \cdot d \vec{x}\right] \quad, \quad a=-\frac{1}{H \eta}
$$

where the $D-1$ spatial coordinates can be any real number $-\infty<x^{i}<+\infty$, but the conformal time is negative definite $-\infty<\eta<0$. Our work is dramatically simplified by conformally rescaling the metric and the scalar,

$$
g_{\mu \nu} \equiv a^{2} \widetilde{g}_{\mu \nu} \equiv a^{2}\left(\eta_{\mu \nu}+\kappa h_{\mu \nu}\right) \quad, \quad \phi \equiv \frac{\widetilde{\phi}}{a^{\frac{D}{2}-1}}
$$

where $\kappa^{2} \equiv 16 \pi G$ and graviton indices are raised and lowered with the Minkowski metric, $h^{\mu}{ }_{\nu} \equiv \eta^{\mu \rho} h_{\rho \nu}$. Up to a surface term the conformal rescaling allows us to express the Lagrangian as,

$$
\begin{array}{r}
\mathcal{L} \longrightarrow \frac{(D-2)}{2} H a^{D-1} \sqrt{-\widetilde{g}} \widetilde{g}^{\rho \sigma} \widetilde{g}^{\mu \nu} h_{\rho \sigma, \mu} h_{\nu 0}+a^{D-2} \sqrt{-\widetilde{g}} \widetilde{g}^{\alpha \beta} \widetilde{g}^{\rho \sigma} \widetilde{g}^{\mu \nu} \\
\times\left\{\frac{1}{2} h_{\alpha \rho, \mu} h_{\nu \sigma, \beta}-\frac{1}{2} h_{\alpha \beta, \rho} h_{\sigma \mu, \nu}+\frac{1}{4} h_{\alpha \beta, \rho} h_{\mu \nu, \sigma}-\frac{1}{4} h_{\alpha \rho, \mu} h_{\beta \sigma, \nu}\right\} \\
-\frac{1}{2} \partial_{\mu} \widetilde{\phi} \partial_{\nu} \widetilde{\phi} \widetilde{g}^{\mu \nu} \sqrt{-\widetilde{g}}-\frac{1}{8}\left(\frac{D-2}{D-1}\right) \widetilde{\phi}^{2} \widetilde{R} \sqrt{-\widetilde{g}} .
\end{array}
$$

Note that $\widetilde{g}^{\alpha \beta}$ and $\widetilde{R}$ are infinite order in the graviton field. 
The single graviton loop contribution to the self-mass of $\tilde{\phi}$ can be expressed in terms of free expectation values of variations of the action,

$$
-i \widetilde{M}^{2}\left(x ; x^{\prime}\right)=\left\langle\Omega\left|T^{*}\left\{\left[\frac{i \delta S}{\delta \widetilde{\phi}(x)}\right]_{h \widetilde{\phi}} \times\left[\frac{i \delta S}{\delta \widetilde{\phi}\left(x^{\prime}\right)}\right]_{h \widetilde{\phi}}+\left[\frac{i \delta^{2} S}{\delta \widetilde{\phi}(x) \delta \widetilde{\phi}\left(x^{\prime}\right)}\right]_{h h}\right\}\right| \Omega\right\rangle,
$$

where the $T^{*}$-ordering symbol indicates that any derivative is acted outside the time-ordered product of the fields. The self-mass of the original field $\phi$ is easy to recover,

$$
-i M^{2}\left(x ; x^{\prime}\right) \equiv\left(a a^{\prime}\right)^{\frac{D}{2}-1} \times-i \widetilde{M}^{2}\left(x ; x^{\prime}\right) .
$$

The first term in (6) corresponds to the left hand diagram of Figure 1 while the second term is represented by the middle diagram.
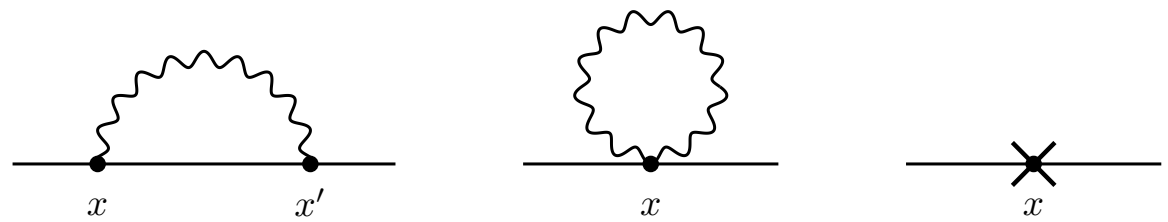

Figure 1: One graviton contributions to $-i M^{2}\left(x ; x^{\prime}\right)$. Graviton lines are wavy and scalar lines are straight. Counterterms are denoted by a cross.

The variations in expression (6) are,

$$
\begin{gathered}
\frac{\delta S}{\delta \widetilde{\phi}(x)}=\partial_{\mu}\left(\sqrt{-\widetilde{g}} \widetilde{g}^{\mu \nu} \partial_{\nu} \widetilde{\phi}\right)-\frac{1}{4}\left(\frac{D-2}{D-1}\right) \widetilde{\phi} \widetilde{R} \sqrt{-\widetilde{g}} \\
\frac{\delta^{2} S}{\delta \widetilde{\phi}(x) \delta \widetilde{\phi}\left(x^{\prime}\right)}=\partial_{\mu}\left(\sqrt{-\widetilde{g}} \widetilde{g}^{\mu \nu} \partial_{\nu} \delta^{D}\left(x-x^{\prime}\right)\right)-\frac{1}{4}\left(\frac{D-2}{D-1}\right) \widetilde{R} \sqrt{-\widetilde{g}} \delta^{D}\left(x-x^{\prime}\right) .
\end{gathered}
$$

It is best to break (8) up into two parts,

$$
\frac{\delta S_{a}}{\delta \widetilde{\phi}(x)}=\partial_{\mu}\left(\sqrt{-\widetilde{g}} \widetilde{g}^{\mu \nu} \partial_{\nu} \widetilde{\phi}\right) \quad, \quad \frac{\delta S_{b}}{\delta \widetilde{\phi}(x)}=-\frac{1}{4}\left(\frac{D-2}{D-1}\right) \widetilde{\phi} \widetilde{R} \sqrt{-\widetilde{g}},
$$

and similarly for the 2 nd variational derivatives. The variation of $S_{b}$ can be usefully further decomposed with the identity,

$$
\begin{aligned}
\sqrt{-\widetilde{g}} \widetilde{R}=\partial_{\rho}\left[\sqrt{-\widetilde{g}}\left(\widetilde{g}^{\mu \nu} \widetilde{\Gamma}_{\mu \nu}^{\rho}-\widetilde{g}^{\rho \mu} \widetilde{\Gamma}_{\nu \mu}^{\nu}\right)\right] & \\
& +\sqrt{-\widetilde{g}} \widetilde{g}^{\mu \nu}\left(\widetilde{\Gamma}_{\sigma \mu}^{\rho} \widetilde{\Gamma}_{\rho \nu}^{\sigma}-\widetilde{\Gamma}_{\rho \sigma}^{\rho} \widetilde{\Gamma}_{\mu \nu}^{\sigma}\right) .
\end{aligned}
$$


Hence we have,

$$
\begin{aligned}
\frac{\delta S_{b 1}}{\delta \widetilde{\phi}(x)} & =-\frac{1}{4}\left(\frac{D-2}{D-1}\right) \widetilde{\phi} \partial_{\rho}\left[\sqrt{-\widetilde{g}}\left(\widetilde{g}^{\mu \nu} \widetilde{\Gamma}_{\mu \nu}^{\rho}-\widetilde{g}^{\rho \mu} \widetilde{\Gamma}_{\nu \mu}^{\nu}\right)\right] \\
\frac{\delta S_{b 2}}{\delta \widetilde{\phi}(x)} & =-\frac{1}{4}\left(\frac{D-2}{D-1}\right) \widetilde{\phi} \sqrt{-\widetilde{g}} \widetilde{g}^{\mu \nu}\left(\widetilde{\Gamma}_{\sigma \mu}^{\rho} \widetilde{\Gamma}_{\rho \nu}^{\sigma}-\widetilde{\Gamma}_{\rho \sigma}^{\rho} \widetilde{\Gamma}_{\mu \nu}^{\sigma}\right) .
\end{aligned}
$$

The expansions of all variations in powers of the gravitons are simplified using the trace-reversed graviton field,

$$
\widehat{h}_{\mu \nu} \equiv h_{\mu \nu}-\frac{1}{2} \eta_{\mu \nu} h
$$

The expansion of the two first variations are,

$$
\begin{aligned}
& {\left[\frac{i \delta S_{a}}{\delta \widetilde{\phi}(x)}\right]_{h \widetilde{\phi}}=-i \kappa \partial_{\mu}\left[\widehat{h}^{\mu \nu} \partial_{\nu} \widetilde{\phi}\right],} \\
& {\left[\frac{i \delta S_{b}}{\delta \widetilde{\phi}(x)}\right]_{h \widetilde{\phi}}=-\frac{i \kappa}{4}\left(\frac{D-2}{D-1}\right) \widetilde{\phi}\left[\partial_{\mu} \partial_{\nu}+\frac{\eta_{\mu \nu} \partial^{2}}{D-2}\right] \widehat{h}^{\mu \nu} .}
\end{aligned}
$$

The second variations are simplest when expressed using both $h_{\mu \nu}$ and $\widehat{h}_{\mu \nu}$,

$$
\begin{aligned}
& {\left[\frac{i \delta^{2} S_{a}}{\delta \widetilde{\phi}(x) \delta \widetilde{\phi}\left(x^{\prime}\right)}\right]_{h h}=i \kappa^{2} \partial_{\mu}\left[\left[\widehat{h}^{\mu \rho} h_{\rho}^{\nu}-\frac{1}{4} \eta^{\mu \nu} \widehat{h}^{\rho \sigma} h_{\rho \sigma}\right] \partial_{\nu} \delta^{D}\left(x-x^{\prime}\right)\right],} \\
& {\left[\frac{i \delta^{2} S_{b 1}}{\left.\delta \widetilde{\phi}(x) \delta \widetilde{\phi}\left(x^{\prime}\right)\right]_{h h}}=\frac{i \kappa^{2}}{4}\left(\frac{D-2}{D-1}\right)\right.} \\
& \quad \times \partial_{\rho}\left[\partial^{\alpha}\left(h^{\rho \beta} \widehat{h}_{\alpha \beta}\right)-\frac{1}{2} \partial^{\rho}\left(h^{\alpha \beta} \widehat{h}_{\alpha \beta}\right)-\frac{1}{2} h^{\rho \sigma} h_{, \sigma}\right] \delta^{D}\left(x-x^{\prime}\right), \\
& {\left[\frac{i \delta^{2} S_{b 2}}{\delta \widetilde{\phi}(x) \delta \widetilde{\phi}\left(x^{\prime}\right)}\right]_{h h}=\frac{i \kappa^{2}}{4}\left(\frac{D-2}{D-1}\right)\left[-\frac{1}{2} \widehat{h}^{\rho \sigma, \mu} \widehat{h}_{\mu \rho, \sigma}+\frac{1}{4} \widehat{h}^{\rho \sigma, \mu} h_{\rho \sigma, \mu}\right] \delta^{D}\left(x-x^{\prime}\right) .}
\end{aligned}
$$

Note that the two graviton fields in each of these expressions are both evaluated at the point $x^{\mu}$.

The propagator of $\widetilde{\phi}$ is the same as for a massless scalar in flat space,

$$
i \Delta\left(x ; x^{\prime}\right)=\frac{\Gamma\left(\frac{D}{2}-1\right)}{4 \pi^{\frac{D}{2}} \Delta x^{D-2}} \quad, \quad \Delta x^{2}\left(x ; x^{\prime}\right) \equiv\left\|\vec{x}-\vec{x}^{\prime}\right\|^{2}-\left(\left|\eta-\eta^{\prime}\right|-i \epsilon\right)^{2} .
$$


The graviton propagator is defined by adding the gauge fixing term [5, 6],

$$
\mathcal{L}_{\mathrm{GF}}=-\frac{1}{2} a^{D-2} \eta^{\mu \nu} F_{\mu} F_{\nu} \quad, \quad F_{\mu}=\eta^{\rho \sigma}\left(h_{\mu \rho, \sigma}-\frac{1}{2} h_{\rho \sigma, \mu}+(D-2) H a h_{\mu \rho} \delta_{\sigma}^{0}\right) .
$$

The graviton propagator in this gauge takes the form,

$$
i\left[{ }_{\mu \nu} \Delta_{\rho \sigma}\right]\left(x ; x^{\prime}\right)=\sum_{I=A, B, C}\left[\mu \nu T_{\rho \sigma}^{I}\right] \times i \Delta_{I}\left(x ; x^{\prime}\right) .
$$

The three propagators $i \Delta_{I}\left(x ; x^{\prime}\right)$ are for minimally coupled scalars with masses $M_{A}^{2}=0, M_{B}^{2}=(D-2) H^{2}$ and $M_{C}^{2}=2(D-3) H^{2}$, so our four scalar propagators obey the equations,

$\partial^{2} i \Delta\left(x ; x^{\prime}\right)=i \delta^{D}\left(x-x^{\prime}\right),\left[\partial^{2}-(D-2) H a \partial_{0}-M_{I}^{2} a^{2}\right] i \Delta_{I}\left(x ; x^{\prime}\right)=\frac{i \delta^{D}\left(x-x^{\prime}\right)}{a^{D-2}}$.

Because $-i \widetilde{M}^{2}\left(x ; x^{\prime}\right)$ is quarticly divergent at one loop we only need the first few terms in the expansions of the three $i \Delta_{I}\left(x ; x^{\prime}\right)$ [5, 6, 40,

$$
\begin{aligned}
& i \Delta_{A}=\frac{i \Delta\left(x ; x^{\prime}\right)}{\left(a a^{\prime}\right)^{\frac{D}{2}-1}}+ \frac{\Gamma\left(\frac{D}{2}+1\right)}{8 \pi^{\frac{D}{2}}(D-4)} \frac{H^{2}}{\left(a a^{\prime} \Delta x^{2}\right)^{\frac{D}{2}-2}}+\frac{\Gamma\left(\frac{D}{2}+2\right)}{64 \pi^{\frac{D}{2}}(D-6)} \frac{H^{4}}{\left(a a^{\prime} \Delta x^{2}\right)^{\frac{D}{2}-3}} \\
&-\frac{H^{D-2}}{(4 \pi)^{\frac{D}{2}}} \frac{\Gamma(D-1)}{\Gamma\left(\frac{D}{2}\right)}\left[\pi \cot \left(\frac{\pi D}{2}\right)-\ln \left(a a^{\prime}\right)\right]+\frac{H^{D}}{(4 \pi)^{\frac{D}{2}}} \frac{\Gamma(D)}{\Gamma\left(\frac{D}{2}+1\right)} \frac{a a^{\prime} \Delta x^{2}}{4}+\cdots,(24) \\
& i \Delta_{B}=\frac{i \Delta\left(x ; x^{\prime}\right)}{\left(a a^{\prime}\right)^{\frac{D}{2}-1}}+ \frac{\Gamma\left(\frac{D}{2}\right)}{16 \pi^{\frac{D}{2}}} \frac{H^{2}}{\left(a a^{\prime} \Delta x^{2}\right)^{\frac{D}{2}-2}}+\frac{\Gamma\left(\frac{D}{2}+1\right)}{128 \pi^{\frac{D}{2}}} \frac{H^{4}}{\left(a a^{\prime} \Delta x^{2}\right)^{\frac{D}{2}-3}} \\
&-\frac{H^{D-2}}{(4 \pi)^{\frac{D}{2}}} \frac{\Gamma(D-2)}{\Gamma\left(\frac{D}{2}\right)}-\frac{H^{D}}{(4 \pi)^{\frac{D}{2}}} \frac{\Gamma(D-1)}{\Gamma\left(\frac{D}{2}+1\right)} \frac{a a^{\prime} \Delta x^{2}}{4}+\cdots, \quad(25) \\
& i \Delta_{C}=\frac{i \Delta\left(x ; x^{\prime}\right)}{\left(a a^{\prime}\right)^{\frac{D}{2}-1}}+ \frac{(D-6) \Gamma\left(\frac{D}{2}-1\right)}{32 \pi^{\frac{D}{2}}} \frac{H^{2}}{\left(a a^{\prime} \Delta x^{2}\right)^{\frac{D}{2}-2}}+\frac{(D-8) \Gamma\left(\frac{D}{2}\right)}{256 \pi^{\frac{D}{2}}} \frac{H^{4}}{\left(a a^{\prime} \Delta x^{2}\right)^{\frac{D}{2}-3}} \\
&+\frac{H^{D-2}}{(4 \pi)^{\frac{D}{2}}} \frac{\Gamma(D-3)}{\Gamma\left(\frac{D}{2}\right)}+\frac{H^{D}}{(4 \pi)^{\frac{D}{2}}} \frac{\Gamma(D-2)}{\Gamma\left(\frac{D}{2}+1\right)} \frac{a a^{\prime} \Delta x^{2}}{2}+\cdots
\end{aligned}
$$

The three tensor factors of (22) are constructed from $\eta_{\mu \nu}, \bar{\eta}_{\mu \nu} \equiv \eta_{\mu \nu}+$ $\delta_{\mu}^{0} \delta^{0}{ }_{\nu}$ and $\delta^{0}{ }_{\mu}$

$$
\begin{aligned}
& {\left[{ }_{\mu \nu} T_{\rho \sigma}^{A}\right]=2 \bar{\eta}_{\mu(\rho} \bar{\eta}_{\sigma) \nu}-\frac{2}{D-3} \bar{\eta}_{\mu \nu} \bar{\eta}_{\rho \sigma} \quad, \quad\left[{ }_{\mu \nu} T_{\rho \sigma}^{B}\right]=-4 \delta^{0}{ }_{(\mu} \bar{\eta}_{\nu)(\rho} \delta_{\sigma)}^{0},} \\
& {\left[{ }_{\mu \nu} T_{\rho \sigma}^{C}\right]=\frac{2 E_{\mu \nu} E_{\rho \sigma}}{(D-2)(D-3)}, \quad E_{\mu \nu} \equiv(D-3) \delta_{\mu}^{0} \delta^{0}{ }_{\nu}+\bar{\eta}_{\mu \nu},}
\end{aligned}
$$


where parenthesized indices are symmetrized. In addition to reducing the number of terms in each interaction, the trace-reversed graviton $\widehat{h}_{\mu \nu}$ has a simpler propagator than $h_{\mu \nu}$. The mixed propagator is,

$$
\begin{aligned}
& i\left[\widehat{\mu \nu} \Delta_{\rho \sigma}\right] \equiv\left[\delta_{\mu}^{\alpha} \delta_{\nu}^{\beta}-\frac{1}{2} \eta_{\mu \nu} \eta^{\alpha \beta}\right] i\left[{ }_{\alpha \beta} \Delta_{\rho \sigma}\right]=2 \eta_{\mu(\rho} \eta_{\sigma) \nu} i \Delta_{A} \\
& +4 \delta_{(\mu}^{0} \bar{\eta}_{\nu)(\rho} \delta_{\sigma)}^{0}\left[i \Delta_{A}-i \Delta_{B}\right]-\frac{2 \delta_{\mu}^{0} \delta_{\nu}^{0} E_{\rho \sigma}}{D-3}\left[i \Delta_{A}-i \Delta_{C}\right] .
\end{aligned}
$$

Note from the propagator expansions (24,26) that the differences $\left[i \Delta_{A}-i \Delta_{B}\right]$ and $\left[i \Delta_{A}-i \Delta_{C}\right]$ are less singular near coincidence than any of the $i \Delta_{I}$. The fully trace-reversed propagator is,

$$
\begin{aligned}
i\left[{ }_{\mu \nu} \Delta_{\widehat{\rho \sigma}}\right] \equiv & {\left[\delta_{\rho}^{\alpha} \delta_{\sigma}^{\beta}-\frac{1}{2} \eta_{\rho \sigma} \eta^{\alpha \beta}\right] i\left[_{\widehat{\mu \nu}} \Delta_{\alpha \beta}\right]=\left[2 \eta_{\mu(\rho} \eta_{\sigma) \nu}-\eta_{\mu \nu} \eta_{\rho \sigma}\right] i \Delta_{A} } \\
& +4 \delta_{(\mu}^{0} \bar{\eta}_{\nu)(\rho} \delta_{\sigma)}^{0}\left[i \Delta_{A}-i \Delta_{B}\right]-2\left(\frac{D-2}{D-3}\right) \delta_{\mu}^{0} \delta_{\nu}^{0} \delta_{\rho}^{0} \delta_{\sigma}^{0}\left[i \Delta_{A}-i \Delta_{C}\right] .
\end{aligned}
$$

\section{Primitive Contributions}

The purpose of this section is to evaluate the left hand and middle diagrams of Figure 1. We first compute the simpler, middle diagram, which is based on the interactions (17, 19). Then we tackle the more difficult, middle diagram which is based on products of the interactions (15, 16). The section closes with a total for all primitive diagrams.

\subsection{4-Point Contributions}

The middle diagram of Figure 1 consists of a single, differentiated and coincident graviton propagator evaluated between the interactions (17, 19). When one takes the coincidence limit before differentiating, the only spacetime dependence is the $\ln (a)$ part of the $i \Delta_{A}$ propagator,

$$
\begin{aligned}
& \left.i \Delta_{A}\left(x ; x^{\prime}\right)\right|_{x^{\prime}=x}=-\frac{H^{D-2}}{(4 \pi)^{\frac{D}{2}}} \frac{\Gamma(D-1)}{\Gamma\left(\frac{D}{2}\right)}\left[\pi \cot \left(\frac{\pi D}{2}\right)-2 \ln (a)\right] \rightarrow-A_{0}+\frac{H^{2} \ln (a)}{4 \pi^{2}}, \\
& \left.i \Delta_{B}\left(x ; x^{\prime}\right)\right|_{x^{\prime}=x}=-\frac{H^{D-2}}{(4 \pi)^{\frac{D}{2}}} \frac{\Gamma(D-2)}{\Gamma\left(\frac{D}{2}\right)} \longrightarrow-\frac{H^{2}}{16 \pi^{2}} \equiv B_{0} \\
& \left.i \Delta_{C}\left(x ; x^{\prime}\right)\right|_{x^{\prime}=x}+\frac{H^{D-2}}{(4 \pi)^{\frac{D}{2}}} \frac{\Gamma(D-3)}{\Gamma\left(\frac{D}{2}\right)} \longrightarrow+\frac{H^{2}}{16 \pi^{2}} \equiv C_{0}
\end{aligned}
$$


Note that only $A_{0}$ is divergent. Taking the coincidence limit after differentiating produces only finite results,

$$
\begin{aligned}
\left.\partial_{\mu} \partial_{\nu}^{\prime} i \Delta_{A}\left(x ; x^{\prime}\right)\right|_{x^{\prime}=x} & =-\frac{1}{2} g_{\mu \nu} \times \frac{H^{D}}{(4 \pi)^{\frac{D}{2}}} \frac{\Gamma(D)}{\Gamma\left(\frac{D}{2}+1\right)} \longrightarrow-\frac{3 H^{4}}{32 \pi^{2}} g_{\mu \nu}, \\
\left.\partial_{\mu} \partial_{\nu}^{\prime} i \Delta_{B}\left(x ; x^{\prime}\right)\right|_{x^{\prime}=x} & =+\frac{1}{2} g_{\mu \nu} \times \frac{H^{D}}{(4 \pi)^{\frac{D}{2}}} \frac{\Gamma(D-1)}{\Gamma\left(\frac{D}{2}+1\right)} \longrightarrow+\frac{H^{4}}{32 \pi^{2}} g_{\mu \nu}, \\
\left.\partial_{\mu} \partial_{\nu}^{\prime} i \Delta_{C}\left(x ; x^{\prime}\right)\right|_{x^{\prime}=x} & =-g_{\mu \nu} \times \frac{H^{D}}{(4 \pi)^{\frac{D}{2}}} \frac{\Gamma(D-2)}{\Gamma\left(\frac{D}{2}+1\right)} \longrightarrow-\frac{H^{4}}{32 \pi^{2}} g_{\mu \nu}
\end{aligned}
$$

\subsection{1 $-i \widetilde{M}_{A}^{2}\left(x ; x^{\prime}\right)$}

What we might call $-i \widetilde{M}_{A}^{2}\left(x ; x^{\prime}\right)$ is the free expectation value of (17),

$$
\begin{array}{r}
-i \widetilde{M}_{A}^{2}=i \kappa^{2} \partial_{\mu}\left\langle\Omega\left|T^{*}\left[\widehat{h}^{\mu \rho}(x) h_{\rho}^{\nu}(x)-\frac{\eta^{\mu \nu}}{4} \widehat{h}^{\rho \sigma}(x) h_{\rho \sigma}(x)\right] \partial_{\nu} \delta^{D}\left(x-x^{\prime}\right)\right| \Omega\right\rangle \\
\left.=i \kappa^{2} \partial_{\mu}\left\{\left[i^{[\widehat{\mu \rho}} \Delta_{\rho}^{\nu}\right](x ; x)-\frac{\eta^{\mu \nu}}{4} i\left[\widehat{\rho \sigma} \Delta_{\rho \sigma}\right](x ; x)\right] \partial_{\nu} \delta^{D}\left(x-x^{\prime}\right)\right\} .
\end{array}
$$

The two contracted propagators we require are,

$$
\begin{aligned}
& i\left[\widehat{\mu \rho} \Delta_{\rho}^{\nu}\right]=\eta^{\mu \nu}\left[(D-1) i \Delta_{B}+2 i \Delta_{C}\right]+\bar{\eta}^{\mu \nu}\left[D i \Delta_{A}-(D-2) i \Delta_{B}-2 i \Delta_{C}\right], \\
& i\left[{ }^{\widehat{\rho \sigma}} \Delta_{\rho \sigma}\right]=D(D-1) i \Delta_{A}+2(D-1) i \Delta_{B}+2 i \Delta_{C} \text {. }
\end{aligned}
$$

Substituting (39-40) in (38) and making use of (31-33) gives,

$-i \widetilde{M}_{A}^{2}\left(x ; x^{\prime}\right)=-\frac{\kappa^{2}}{4} D(D-1) A_{0} \partial \cdot \partial^{\prime}\left[\frac{i \delta^{D}\left(x-x^{\prime}\right)}{\left(a a^{\prime}\right)^{\frac{D}{2}-2}}\right]+\kappa^{2} D A_{0} \vec{\nabla} \cdot \vec{\nabla}^{\prime}\left[\frac{i \delta^{D}\left(x-x^{\prime}\right)}{\left(a a^{\prime}\right)^{\frac{D}{2}-2}}\right]$.

\subsection{2 $-i \widetilde{M}_{B}^{2}\left(x ; x^{\prime}\right)$}

What should be called $-i \widetilde{M}_{B 1}^{2}\left(x ; x^{\prime}\right)$ is the free expectation value of (18),

$$
\begin{array}{r}
-i \widetilde{M}_{B 1}^{2} \equiv\left\langle\Omega\left|T^{*}\left[\frac{i \delta^{2} S_{b 1}}{\delta \widetilde{\phi}(x) \delta \widetilde{\phi}\left(x^{\prime}\right)}\right]_{h h}\right| \Omega\right\rangle=\frac{\kappa^{2}}{4}\left(\frac{D-2}{D-1}\right) \partial_{\rho}\left[\partial^{\sigma} i\left[{ }^{\rho \beta} \Delta_{\sigma \beta}\right](x ; x)\right. \\
\left.-\frac{1}{2} \partial^{\rho} i\left[{ }^{\alpha \beta} \Delta_{\widehat{\alpha \beta}}\right](x ; x)-\left.\frac{1}{2} \partial_{\sigma}^{\prime} i\left[{ }^{\rho \sigma} \Delta_{\alpha}^{\alpha}\right]\left(x ; x^{\prime}\right)\right|_{x^{\prime}=x}\right] i \delta^{D}\left(x-x^{\prime}\right) .
\end{array}
$$


Because the three propagators inside the square bracket of (42) are singly differentiated, only the $\ln (a)$ part of the $i \Delta_{A}$ can contribute, and only when differentiated with respect to time. Hence the first and the third propagators give nothing, and the contribution from the second propagator is finite,

$$
-i \widetilde{M}_{B 1}^{2}\left(x ; x^{\prime}\right)=\frac{\kappa^{2} H^{4} a^{2}}{4 \pi^{2}} i \delta^{4}\left(x-x^{\prime}\right) .
$$

The second 4-point contribution is,

$$
\begin{aligned}
& -i \widetilde{M}_{B 2}^{2}\left(x ; x^{\prime}\right) \equiv\left\langle\Omega\left|T^{*}\left[\frac{i \delta^{2} S_{b 1}}{\delta \widetilde{\phi}(x) \delta \widetilde{\phi}\left(x^{\prime}\right)}\right]_{h h}\right| \Omega\right\rangle \\
& =\frac{\kappa^{2}}{8}\left(\frac{D-2}{D-1}\right)\left[-\partial^{\mu} \partial_{\sigma}^{\prime} i\left[\widehat{\rho \sigma} \Delta_{\widehat{\mu \rho}}\right]\left(x ; x^{\prime}\right)+\frac{1}{2} \partial \cdot \partial^{\prime} i\left[\widehat{\rho \sigma}^{\rho} \Delta_{\rho \sigma}\right]\left(x ; x^{\prime}\right)\right] i \delta^{D}\left(x-x^{\prime}\right) .
\end{aligned}
$$

By virtue of the delta function, each of the two propagators in (44) is finite and consists of a sum of the three terms (34) mine the coefficients. Those of the second propagator come from expression (40), and the first propagator derives from a single contraction of (30),

$$
\begin{aligned}
i\left[\widehat{\rho \sigma}_{\widehat{\mu \rho}}\right]=\delta_{\mu}^{\sigma}\left[-\left(\frac{D-1}{D-3}\right)\right. & \left.i \Delta_{A}+(D-1) i \Delta_{B}+2\left(\frac{D-2}{D-3}\right) i \Delta_{C}\right] \\
& +\bar{\delta}_{\mu}^{\sigma}(D-2)\left[\left(\frac{D-1}{D-3}\right) i \Delta_{A}-i \Delta_{B}-\frac{2}{D-3} i \Delta_{C}\right] .
\end{aligned}
$$

Combining everything gives,

$$
-i \widetilde{M}_{B 2}^{2}\left(x ; x^{\prime}\right)=-\frac{\kappa^{2} H^{4} a^{2}}{8 \pi^{2}} i \delta^{4}\left(x-x^{\prime}\right) .
$$

\subsection{3-Point Contributions}

The left hand diagram of Figure 1 represent the free expectation values of product of first variations (15-16) at points $x^{\mu}$ and $x^{\prime \mu}$. Each term involves the product of a (possibly differentiated) graviton propagator multiplied by a (possibly differentiated) scalar propagator. The first and second derivatives of the scalar propagator are,

$$
\begin{aligned}
\partial_{\mu} i \Delta\left(x ; x^{\prime}\right) & =-\frac{\Gamma\left(\frac{D}{2}\right)}{2 \pi^{\frac{D}{2}}} \frac{\Delta x_{\mu}}{\Delta x^{D}} \\
\partial_{\mu} \partial_{\rho}^{\prime} i \Delta\left(x ; x^{\prime}\right) & =\delta_{\mu}^{0} \delta_{\rho}^{0} i \delta^{D}\left(x-x^{\prime}\right)+\frac{\Gamma\left(\frac{D}{2}\right)}{2 \pi^{\frac{D}{2}}}\left[\frac{\eta_{\mu \rho}}{\Delta x^{D}}-\frac{D \Delta x_{\mu} \Delta x_{\rho}}{\Delta x^{D+2}}\right] .
\end{aligned}
$$


Note that the action of two time derivatives of either propagator will produce a delta function, which evaluates the other propagator at coincidence. We have already reviewed the coincidence limits (31-33) of the components of the graviton propagator. A key point about the scalar propagator is that its coincidence limit, and that of its first derivative, both vanish in dimensional regularization. Note also that some of the expressions in this subsection were checked using the symbolic manipulation program "Cadabra" [41,42,

\subsection{1 $-i \widetilde{M}_{a a}^{2}\left(x ; x^{\prime}\right)$}

The free expectation value of the product of two factors of (15) is,

$$
\begin{aligned}
-i \widetilde{M}_{a a}^{2}\left(x ; x^{\prime}\right) & \equiv-\kappa^{2} \partial_{\mu} \partial_{\rho}^{\prime}\left\langle\Omega\left|T^{*}\left[\widehat{h}^{\mu \nu}(x) \partial_{\nu} \widetilde{\phi}(x) \times \widehat{h}^{\rho \sigma}\left(x^{\prime}\right) \partial_{\sigma}^{\prime} \widetilde{\phi}\left(x^{\prime}\right)\right]\right| \Omega\right\rangle, \\
& =-\kappa^{2} \partial_{\mu} \partial_{\rho}^{\prime}\left\{i\left[{ }^{\mu \nu} \Delta^{\widehat{\rho \sigma}}\right]\left(x ; x^{\prime}\right) \times \partial_{\nu} \partial_{\sigma}^{\prime} i \Delta\left(x ; x^{\prime}\right)\right\}
\end{aligned}
$$

Performing the inner contractions over $\nu$ and $\sigma$ gives,

$$
\begin{aligned}
& -i \widetilde{M}_{a a}^{2}\left(x ; x^{\prime}\right)=-\kappa^{2} \partial_{\mu} \partial_{\rho}^{\prime}\left\{i \Delta_{A} \eta^{\mu \rho} \partial \cdot \partial^{\prime} i \Delta+\left(i \Delta_{A}-i \Delta_{B}\right)\left[\delta_{0}^{\mu} \delta_{0}^{\rho} \nabla \cdot \nabla^{\prime}\right.\right. \\
& \left.\left.+\delta_{0}^{\mu} \bar{\partial}^{\rho} \partial_{0}^{\prime}+\delta_{0}^{\rho} \bar{\partial}^{\prime \mu} \partial_{0}+\bar{\eta}^{\mu \rho} \partial_{0} \partial_{0}^{\prime}\right] i \Delta-2\left(\frac{D-2}{D-3}\right)\left(i \Delta_{A}-i \Delta_{C}\right) \delta_{0}^{\mu} \delta_{0}^{\rho} \partial_{0} \partial_{0}^{\prime} i \Delta\right\} .
\end{aligned}
$$

Because the scalar propagator (unlike the graviton propagator!) depends only on the coordinate difference $\left(x-x^{\prime}\right)^{\mu}$, we can convert primed to unprimed derivatives $\partial_{\mu}^{\prime}=-\partial_{\mu}$. We can also use the propagator equation (23) to eliminate double time derivatives,

$$
\partial_{0} \partial_{0}^{\prime} i \Delta\left(x ; x^{\prime}\right)=i \delta^{D}\left(x-x^{\prime}\right)-\nabla^{2} i \Delta\left(x ; x^{\prime}\right) .
$$

These reductions uncover a local part and a nonlocal one,

$$
\begin{array}{r}
-i \widetilde{M}_{a a}^{2}\left(x ; x^{\prime}\right)=\kappa^{2} \partial_{\mu} \partial_{\rho}^{\prime}\left\{\left[\eta^{\mu \rho} i \Delta_{A}-\bar{\eta}^{\mu \rho}\left(i \Delta_{A}-i \Delta_{B}\right)+2\left(\frac{D-2}{D-3}\right) \delta_{0}^{\mu} \delta_{0}^{\rho}\right.\right. \\
\left.\times\left(i \Delta_{A}-i \Delta_{C}\right)\right] i \delta^{D}\left(x-x^{\prime}\right)+\left(i \Delta_{A}-i \Delta_{B}\right)\left[\left(\delta_{0}^{\mu} \delta_{0}^{\rho}+\bar{\eta}^{\mu \rho}\right) \nabla^{2}+2 \delta_{0}^{(\mu} \bar{\partial}^{\rho)} \partial_{0}\right] i \Delta \\
\left.-2\left(\frac{D-2}{D-3}\right)\left(i \Delta_{A}-i \Delta_{C}\right) \delta_{0}^{\mu} \delta_{0}^{\rho} \nabla^{2} i \Delta\right\} .
\end{array}
$$


Further reducing the local contributions of (53) is easy. The coincident propagators can be read off from expressions (31]+33), and the three tensor structures can be consolidated to two using $\delta_{0}^{\mu} \delta_{0}^{\rho}=\bar{\eta}^{\mu \rho}-\eta^{\mu \rho}$. The three nonlocal contributions of (53) require more work. We first extract derivatives using the relations,

$$
\begin{aligned}
\partial_{0} \partial_{i}\left(\frac{1}{\Delta x^{D-2}}\right) \times \frac{1}{\Delta x^{D-4}} & =\frac{1}{4}\left(\frac{D}{D-3}\right) \partial_{0} \partial_{i}\left(\frac{1}{\Delta x^{2 D-6}}\right), \\
\nabla^{2}\left(\frac{1}{\Delta x^{D-2}}\right) \times \frac{1}{\Delta x^{D-4}} & =\frac{1}{4}\left[\left(\frac{D}{D-3}\right) \nabla^{2}-\left(\frac{D-1}{D-3}\right) \partial^{2}\right] \frac{1}{\Delta x^{2 D-6}},
\end{aligned}
$$

We also need the identity [43],

$$
\partial^{2} \frac{1}{\Delta x^{2 D-6}}=\frac{\mu^{D-4} 4 \pi^{\frac{D}{2}} i \delta^{D}\left(x-x^{\prime}\right)}{\Gamma\left(\frac{D}{2}-1\right)}-\frac{1}{2}(D-4) \partial^{2}\left(\frac{\ln \left(\mu^{2} \Delta x^{2}\right)}{\Delta x^{2}}\right)+O\left((D-4)^{2}\right) .
$$

Relations (54 (56) imply,

$$
\begin{aligned}
&\left(i \Delta_{A}-i \Delta_{B}\right) \partial_{0} \partial_{i} i \Delta=-\frac{H^{2} \partial_{0} \partial_{i}}{16 \pi^{4}}\left\{\frac{\frac{1}{2} \ln \left(\frac{1}{4} H^{2} \Delta x^{2}\right)+1}{\Delta x^{2}}\right\} \\
&\left(i \Delta_{A}-i \Delta_{B}\right) \nabla^{2} i \Delta=-\frac{H^{2} \nabla^{2}}{16 \pi^{4}}\left\{\frac{\frac{1}{2} \ln \left(\frac{1}{4} H^{2} \Delta x^{2}\right)+1}{\Delta x^{2}}\right\}+\frac{3 H^{2} \partial^{2}}{128 \pi^{4}}\left\{\frac{\ln \left(\mu^{2} \Delta x^{2}\right)}{\Delta x^{2}}\right\} \\
&-\frac{\mu^{D-4} H^{2}}{16 \pi^{\frac{D}{2}}} \frac{(D-1) \Gamma\left(\frac{D}{2}\right)}{(D-3)(D-4)} \frac{i \delta^{D}\left(x-x^{\prime}\right)}{\left(a a^{\prime}\right)^{\frac{D}{2}-2}},(58) \\
&\left(i \Delta_{A}-i \Delta_{C}\right) \nabla^{2} i \Delta=-\frac{H^{2} \nabla^{2}}{16 \pi^{4}}\left\{\frac{\frac{1}{2} \ln \left(\frac{1}{4} H^{2} \Delta x^{2}\right)+1}{\Delta x^{2}}\right\}+\frac{3 H^{2} \partial^{2}}{128 \pi^{4}}\left\{\frac{\ln \left(\mu^{2} \Delta x^{2}\right)}{\Delta x^{2}}\right\} \\
&-\frac{\mu^{D-4} H^{2}}{16 \pi^{\frac{D}{2}}} \frac{2(D-1) \Gamma\left(\frac{D}{2}\right)}{(D-2)(D-4)} \frac{i \delta^{D}\left(x-x^{\prime}\right)}{\left(a a^{\prime}\right)^{\frac{D}{2}-2}},
\end{aligned}
$$

where we have ignored terms that vanish at $D=4$. Putting everything together gives,

$$
\begin{aligned}
-i \widetilde{M}_{a a}^{2}\left(x ; x^{\prime}\right)= & \kappa^{2}\left[\left(\frac{D-1}{D-3}\right)\left(A_{0}-\frac{3}{4} A_{1}\right)+\frac{H^{2}}{4 \pi^{2}}\right] \partial \cdot \partial^{\prime}\left\{\frac{i \delta^{D}\left(x-x^{\prime}\right)}{\left(a a^{\prime}\right)^{\frac{D}{2}-2}}\right\} \\
& -\kappa^{2}\left[\left(\frac{D-1}{D-3}\right)\left(A_{0}-\frac{1}{2} A_{1}\right)+\frac{5 H^{2}}{16 \pi^{2}}\right] \vec{\nabla} \cdot \vec{\nabla}^{\prime}\left\{\frac{i \delta^{D}\left(x-x^{\prime}\right)}{\left(a a^{\prime}\right)^{\frac{D}{2}-2}}\right\} \\
+ & \frac{\kappa^{2} H^{2} \partial^{2} \nabla^{2}}{16 \pi^{4}}\left\{\frac{\frac{1}{2} \ln \left(\frac{1}{4} H^{2} \Delta x^{2}\right)+1}{\Delta x^{2}}\right\}-\frac{3 \kappa^{2} H^{2} \partial^{2}\left(3 \partial^{2}-2 \nabla^{2}\right)}{128 \pi^{4}}\left\{\frac{\ln \left(\mu^{2} \Delta x^{2}\right)}{\Delta x^{2}}\right\} .
\end{aligned}
$$


where the constants $A_{0}$ and $A_{1}$ are,

$$
A_{0} \equiv \frac{H^{D-2}}{(4 \pi)^{\frac{D}{2}}} \frac{\Gamma(D-1)}{\Gamma\left(\frac{D}{2}\right)} \times \pi \cot \left(\frac{\pi D}{2}\right) \quad, \quad A_{1} \equiv \frac{\mu^{D-4} H^{2}}{4 \pi^{\frac{D}{2}}} \frac{\Gamma\left(\frac{D}{2}\right)}{D-4}
$$

\subsection{2 $-i \widetilde{M}_{a b}^{2}\left(x ; x^{\prime}\right)$ and $-i \widetilde{M}_{b a}^{2}\left(x ; x^{\prime}\right)$}

Although there are two products of (15) and (16), we first consider the product of (15) at $x^{\mu}$ with (16) at $x^{\prime \mu}$, and then derive the crossed contribution by interchanging the coordinates,

$$
\begin{gathered}
-i \widetilde{M}_{a b}^{2}\left(x ; x^{\prime}\right) \\
\equiv-\frac{\kappa^{2}}{4}\left(\frac{D-2}{D-1}\right) \partial_{\mu}\left\langle\Omega\left|T^{*}\left[\widehat{h}^{\mu \nu}(x) \partial_{\nu} \widetilde{\phi}(x) \times \widetilde{\phi}\left(x^{\prime}\right)\left[\partial_{\rho}^{\prime} \partial_{\sigma}^{\prime}+\frac{\eta_{\rho \sigma} \partial^{\prime 2}}{D-2}\right] \widehat{h}^{\rho \sigma}\left(x^{\prime}\right)\right]\right| \Omega\right\rangle, \\
=-\frac{\kappa^{2}}{4}\left(\frac{D-2}{D-1}\right) \partial_{\mu}\left\{\partial_{\nu} i \Delta\left(x ; x^{\prime}\right) \times\left[\partial_{\rho}^{\prime} \partial_{\sigma}^{\prime}+\frac{\eta_{\rho \sigma} \partial^{\prime 2}}{D-2}\right] i\left[\widehat{\mu \nu} \Delta^{\rho \sigma}\right]\left(x ; x^{\prime}\right)\right\} \\
=\frac{\kappa^{2}}{2}\left(\frac{D-2}{D-1}\right) \partial_{\mu}\left\{\partial _ { \nu } i \Delta \left[\left(\eta^{\mu \nu} \partial^{\prime 2}-\partial^{\prime \mu} \partial^{\prime \nu}\right) i \Delta_{A}-2 \delta_{0}^{(\mu} \bar{\partial}^{\prime \nu)} \partial_{0}^{\prime}\left[i \Delta_{A}-i \Delta_{B}\right]\right.\right. \\
\left.\left.+\frac{\delta_{0}^{\mu} \delta_{0}^{\nu}}{D-3}\left[-(D-1) \partial^{\prime 2}+(D-2) \nabla^{\prime 2}\right]\left[i \Delta_{A}-i \Delta_{C}\right]\right]\right\}
\end{gathered}
$$

If we keep the $\partial_{\mu}$ derivative outside the curly brackets the terms inside diverge only cubicly, which reduces the number of terms that must be retained in the expansion of the three $i \Delta_{I}\left(x ; x^{\prime}\right)$. Our strategy is accordingly to extract derivatives from the curly brackets.

The three tensor factors inside the large square brackets of (64) have the $3+1$ decompositions,

$$
\begin{aligned}
\left(\eta^{\mu \nu} \partial^{\prime 2}-\partial^{\prime \mu} \partial^{\prime \nu}\right) & =\left(\begin{array}{cc}
-\nabla^{\prime 2} & \partial_{0}^{\prime} \partial_{n}^{\prime} \\
\partial_{m}^{\prime} \partial_{0}^{\prime} & \delta_{m n} \partial^{\prime 2}-\partial_{m}^{\prime} \partial_{n}^{\prime}
\end{array}\right) \\
\left(\delta_{0}^{\mu} \bar{\partial}^{\prime \nu}+\delta_{0}^{\nu} \bar{\partial}^{\prime \mu}\right) \partial_{0}^{\prime} & =\left(\begin{array}{cc}
0 & \partial_{0}^{\prime} \partial_{n}^{\prime} \\
\partial_{m}^{\prime} \partial_{0}^{\prime} & 0
\end{array}\right) \\
\delta_{0}^{\mu} \delta_{0}^{\nu} & =\left(\begin{array}{cc}
1 & 0 \\
0 & 0
\end{array}\right) .
\end{aligned}
$$

We next use the propagator equations (23), and the fact that the coincidence limit of $\partial_{\nu} i \Delta\left(x ; x^{\prime}\right)$ vanishes, to contract the factor inside the large square 
brackets of expression (64) into the derivative of the scalar. The results are,

$$
\begin{aligned}
& {[]^{0 \nu} \partial_{\nu} i \Delta=\left[-\left[\nabla^{\prime 2}-2(D-1) H^{2} a^{\prime 2}\right] i \Delta_{C}\right.} \\
& \left.+\left[\nabla^{\prime 2}-(D-2)(D-1) H a^{\prime} \partial_{0}^{\prime}\right]\left(\frac{i \Delta_{A}-i \Delta_{C}}{D-3}\right)\right] \partial_{0} i \Delta+\partial_{0}^{\prime} \partial_{n}^{\prime} i \Delta_{B} \partial_{n} i \Delta, \\
& {[\quad]^{m \nu} \partial_{\nu} i \Delta=\partial_{0}^{\prime} \partial_{m}^{\prime} i \Delta_{B} \partial_{0} i \Delta+\left[\delta_{m n}(D-2) H a^{\prime} \partial_{0}^{\prime}-\partial_{m}^{\prime} \partial_{n}^{\prime}\right] i \Delta_{A} \partial_{n} i \Delta .}
\end{aligned}
$$

The next step is to act the derivatives in expressions (68, 69), combine terms and extract derivatives. These manipulations are very tedious so we only present the results, dropping terms that vanish for $D=4$,

$$
\begin{aligned}
& {[]_{\nu}^{0 \nu} \partial_{\nu} i \Delta=\frac{\Gamma^{2}\left(\frac{D}{2}\right) \partial_{0}}{8 \pi^{D}}\left\{\frac{\partial^{\prime 2}}{2(D-2)^{2}}\left[\frac{1}{\left(a a^{\prime} \Delta x^{4}\right)^{\frac{D}{2}-1}}\right]+\frac{\left(D^{2}+10 D-8\right) H^{2} a^{\prime 2}}{8(D-2)^{2}\left(a a^{\prime} \Delta x^{4}\right)^{\frac{D}{2}-1}}\right.} \\
& -\frac{1}{2}\left(\frac{3 D-4}{D-1}\right) \frac{H a^{\prime} \Delta x_{0}}{\left(a a^{\prime}\right)^{\frac{D}{2}-1} \Delta x^{2 D-2}}+\frac{1}{2}\left(\frac{D-2}{D-1}\right) \frac{H^{2} \Delta x_{0}^{2}}{\left(a a^{\prime}\right)^{\frac{D}{2}-2} \Delta x^{2 D-2}} \\
& \left.-\frac{(D+1)\left(D^{2}-7 D+8\right) H^{2}}{8(D-1)(D-2)\left(a a^{\prime}\right)^{\frac{D}{2}-2} \Delta x^{2 D-4}}-\left(\frac{D-1}{D-2}\right) \frac{H^{3} a^{\prime} \Delta x_{0}}{\left(a a^{\prime}\right)^{\frac{D}{2}-2} \Delta x^{2 D-4}}\right\} \\
& +\frac{\Gamma^{2}\left(\frac{D}{2}\right)}{8 \pi^{D}}\left\{\frac{1}{2}\left(\frac{D-2}{D-1}\right) \frac{\left[(D-1) H a+(2 D-3) H a^{\prime}-\frac{1}{2}(D-2) H^{2} a a^{\prime} \Delta x_{0}\right]}{\left(a a^{\prime}\right)^{\frac{D}{2}-1} \Delta x^{2 D-2}}\right. \\
& +\frac{(D-4) H^{2}}{4\left(a a^{\prime}\right)^{\frac{D}{2}-2}}\left[\left(\frac{D-2}{D-1}\right) \frac{H a \Delta x_{0}^{2}}{\Delta x^{2 D-2}}+\frac{\left(D^{2}-7 D+8\right)(D+1)}{4(D-2)(D-1)} \frac{H a}{\Delta x^{2 D-4}}\right. \\
& \left.\left.-2\left(\frac{D-1}{D-2}\right) \frac{H^{2} a a^{\prime} \Delta x_{0}}{\Delta x^{2 D-4}}\right]\right\} \\
& {[]^{m \nu} \partial_{\nu} i \Delta=\frac{\Gamma^{2}\left(\frac{D}{2}\right) \partial_{m}}{8 \pi^{D}}\left\{\frac{-\partial^{\prime 2}}{2(D-2)^{2}}\left[\frac{1}{\left(a a^{\prime} \Delta x^{4}\right)^{\frac{D}{2}-1}}\right]-\frac{(5 D-12) H^{2} a^{\prime 2}}{8(D-2)\left(a a^{\prime} \Delta x^{4}\right)^{\frac{D}{2}-1}}\right.} \\
& +\frac{1}{2}\left(\frac{3 D-4}{D-1}\right) \frac{H a^{\prime} \Delta x_{0}}{\left(a a^{\prime}\right)^{\frac{D}{2}-1} \Delta x^{2 D-2}}-\frac{1}{2}\left(\frac{D-2}{D-1}\right) \frac{H^{2} \Delta x_{0}^{2}}{\left(a a^{\prime}\right)^{\frac{D}{2}-2} \Delta x^{2 D-2}} \\
& \left.+\frac{D H^{2}\left[-(D-3)+(D-2) H a^{\prime} \Delta x_{0}\right]}{8(D-2)\left(a a^{\prime}\right)^{\frac{D}{2}-2} \Delta x^{2 D-4}}\right\} \text {. }
\end{aligned}
$$

We now substitute expressions (70-71) in (64) and extract derivatives from each term until the expression becomes integrable. At that point divergences are localized and the unregulated limit is taken on the nonlocal 
terms. Relevant identities are,

$$
\begin{aligned}
& \frac{1}{\Delta x^{2 D-2}} \longrightarrow \frac{K \partial^{2} i \delta^{D}\left(x-x^{\prime}\right)}{2(D-2)^{2}}-\frac{\partial^{4}}{32}\left[\frac{\ln \left(\mu^{2} \Delta x^{2}\right)}{\Delta x^{2}}\right] \\
& \frac{\Delta x_{0}}{\Delta x^{2 D-2}} \longrightarrow-\frac{K \partial_{0} i \delta^{D}\left(x-x^{\prime}\right)}{2(D-2)}+\frac{\partial_{0} \partial^{2}}{16}\left[\frac{\ln \left(\mu^{2} \Delta x^{2}\right)}{\Delta x^{2}}\right] \\
& \frac{\Delta x_{0}^{2}}{\Delta x^{2 D-2}} \longrightarrow-\frac{K i \delta^{D}\left(x-x^{\prime}\right)}{2(D-2)}+\frac{\partial^{2}}{16}\left[\frac{\ln \left(\mu^{2} \Delta x^{2}\right)-2}{\Delta x^{2}}\right]+\frac{\nabla^{2}}{8}\left[\frac{1}{\Delta x^{2}}\right] \\
& \frac{1}{\Delta x^{2 D-4}} \longrightarrow K i \delta^{D}\left(x-x^{\prime}\right)-\frac{\partial^{2}}{4}\left[\frac{\ln \left(\mu^{2} \Delta x^{2}\right)}{\Delta x^{2}}\right] \\
& \frac{\Delta x_{0}}{\Delta x^{2 D-4}} \longrightarrow-\frac{\partial_{0}}{2}\left[\frac{1}{\Delta x^{2}}\right]
\end{aligned}
$$

where the constant $K$ is,

$$
K \equiv \frac{\mu^{D-4} 4 \pi^{\frac{D}{2}}}{2(D-3)(D-4) \Gamma\left(\frac{D}{2}-1\right)} .
$$

The final result is,

$$
\begin{aligned}
&-i \widetilde{M}_{a b}^{2}\left(x ; x^{\prime}\right)= \frac{-\kappa^{2} A_{1}}{(D-1)(D-3)}\left\{\frac{\partial^{2} \partial^{\prime 2}}{8 H^{2}}\left[\frac{i \delta^{D}\left(x-x^{\prime}\right)}{\left(a a^{\prime}\right)^{\frac{D}{2}-1}}\right]+\frac{3 \partial^{2}}{D-1}\left[\frac{i \delta^{D}\left(x-x^{\prime}\right)}{\left(a a^{\prime}\right)^{\frac{D}{2}-2}}\right]\right\} \\
& \quad+\frac{\kappa^{2} H^{2}}{24 \pi^{2}}\left\{-\frac{11}{6} \nabla^{2}-\frac{17}{12} \partial^{2}-\frac{1}{2} H a^{\prime} \partial_{0}\right\} i \delta^{4}\left(x-x^{\prime}\right) \\
&+\frac{\kappa^{2} H^{2}}{96 \pi^{4}}\left\{\frac{\partial^{2} \partial^{\prime 2}}{8 H^{2}}\left(\frac{1}{a a^{\prime}} \partial^{2}\left[\frac{\ln \left(\mu^{2} \Delta x^{2}\right)}{\Delta x^{2}}\right]\right)+\partial^{2} \partial^{\prime 2}\left[\frac{\ln \left(\mu^{2} \Delta x^{2}\right)}{\Delta x^{2}}\right]\right\} .
\end{aligned}
$$

The result for $-i \widetilde{M}_{b a}^{2}\left(x ; x^{\prime}\right)$ follows by simply interchanging $x^{\mu}$ and $x^{\prime \mu}$. Most of the terms in (78) are already reflection symmetric, and a few simple reductions suffice for those which are not,

$$
\begin{aligned}
& \frac{3 A_{1}\left(\partial^{2}+\partial^{\prime 2}\right)}{(D-1)^{2}(D-3)}\left[\frac{i \delta^{D}\left(x-x^{\prime}\right)}{\left(a a^{\prime}\right)^{\frac{D}{2}-2}}\right] \longrightarrow-\frac{6 A_{1} \partial \cdot \partial^{\prime}}{(D-1)^{2}(D-3)}\left[\frac{i \delta^{D}\left(x-x^{\prime}\right)}{\left(a a^{\prime}\right)^{\frac{D}{2}-2}}\right] \\
&+\frac{H^{4} a^{2} i \delta^{4}\left(x-x^{\prime}\right)}{12 \pi^{2}}, \\
&-\frac{1}{2} H\left(a^{\prime} \partial_{0}+a \partial_{0}^{\prime}\right) i \delta^{4}\left(x-x^{\prime}\right)=-\frac{1}{2} H^{2} a^{2} i \delta^{4}\left(x-x^{\prime}\right) .
\end{aligned}
$$


The final result for sum is,

$$
\begin{aligned}
-i \widetilde{M}_{a b+b a}^{2}= & \frac{\kappa^{2} A_{1}}{(D-1)(D-3)}\left\{-\frac{\partial^{2} \partial^{\prime 2}}{4 H^{2}}\left[\frac{i \delta^{D}\left(x-x^{\prime}\right)}{\left(a a^{\prime}\right)^{\frac{D}{2}-1}}\right]+\frac{6 \partial \cdot \partial^{\prime}}{D-1}\left[\frac{i \delta^{D}\left(x-x^{\prime}\right)}{\left(a a^{\prime}\right)^{\frac{D}{2}-2}}\right]\right\} \\
& +\frac{\kappa^{2} H^{2}}{24 \pi^{2}}\left\{-\frac{11}{3} \nabla^{2}-\frac{17}{6} \partial^{2}-\frac{5}{2} H^{2} a^{2}\right\} i \delta^{4}\left(x-x^{\prime}\right) \\
+ & \frac{\kappa^{2} H^{2}}{96 \pi^{4}}\left\{\frac{\partial^{2} \partial^{\prime 2}}{4 H^{2}}\left(\frac{1}{a a^{\prime}} \partial^{2}\left[\frac{\ln \left(\mu^{2} \Delta x^{2}\right)}{\Delta x^{2}}\right]\right)+2 \partial^{2} \partial^{\prime 2}\left[\frac{\ln \left(\mu^{2} \Delta x^{2}\right)}{\Delta x^{2}}\right]\right\} .
\end{aligned}
$$

\subsection{3 -i $\widetilde{M}_{b b}^{2}\left(x ; x^{\prime}\right)$}

The last of the 3-point contributions is the free expectation value of the product of two (16) interactions. We begin by reducing this to propagators and performing the initial contractions,

$$
\begin{gathered}
-i \widetilde{M}_{b b}^{2}\left(x ; x^{\prime}\right) \equiv-\frac{\kappa^{2}}{16}\left(\frac{D-2}{D-1}\right)^{2} \\
\times\left\langle\Omega\left|T^{*}\left[\widetilde{\phi}(x)\left[\partial_{\mu} \partial_{\nu}+\frac{\eta_{\mu \nu} \partial^{2}}{D-2}\right] \widehat{h}^{\mu \nu}(x) \times \widetilde{\phi}\left(x^{\prime}\right)\left[\partial_{\rho}^{\prime} \partial_{\sigma}^{\prime}+\frac{\eta_{\rho \sigma} \partial^{\prime 2}}{D-2}\right] \widehat{h}^{\rho \sigma}\left(x^{\prime}\right)\right]\right| \Omega\right\rangle \\
=-\frac{\kappa^{2}}{16}\left(\frac{D-2}{D-1}\right)^{2} i \Delta\left(x ; x^{\prime}\right)\left[\partial_{\mu} \partial_{\nu}+\frac{\eta_{\mu \nu} \partial^{2}}{D-2}\right]\left[\partial_{\rho}^{\prime} \partial_{\sigma}^{\prime}+\frac{\eta_{\rho \sigma} \partial^{\prime 2}}{D-2}\right] i\left[\widehat{\mu \nu} \Delta^{\widehat{\rho \sigma}}\right]\left(x ; x^{\prime}\right), \\
=-\frac{\kappa^{2}}{16}\left(\frac{D-2}{D-1}\right)^{2} i \Delta\left\{\left[2\left(\partial \cdot \partial^{\prime}\right)^{2}-2\left(\frac{2 D-3}{D-2}\right) \partial^{2} \partial^{\prime 2}\right] i \Delta_{A}\right. \\
\quad+4 \partial_{0} \partial_{0}^{\prime} \vec{\nabla} \cdot \vec{\nabla}^{\prime}\left(i \Delta_{A}-i \Delta_{B}\right)-\frac{2}{D-3}\left[\frac{(D-1)^{2}}{(D-2)} \partial^{2} \partial^{\prime 2}-(D-1) \partial^{2} \nabla^{\prime 2}\right. \\
\left.\left.\quad-(D-1) \partial^{\prime 2} \nabla^{2}+(D-2) \nabla^{2} \nabla^{\prime 2}\right]\left(i \Delta_{A}-i \Delta_{C}\right)\right\}
\end{gathered}
$$

Because this contribution begins with no derivatives extracted, it is necessary to retain up to second order terms in the propagator expansions (24-26).

At this stage our strategy is to eliminate factors of $\partial^{2}$ and $\partial^{\prime 2}$ using the propagator equations (23). We can also eliminate factors of $\partial_{0} \partial_{0}^{\prime}$ by employing the reflection identities [31,44, 45],

$$
\begin{aligned}
\partial_{0} i \Delta_{A}\left(x ; x^{\prime}\right) & =-\left[\partial_{0}^{\prime}+(D-2) H a^{\prime}\right] i \Delta_{B}\left(x ; x^{\prime}\right), \\
\left(\partial_{0}+H a\right) i \Delta_{B}\left(x ; x^{\prime}\right) & =-\left[\partial_{0}^{\prime}+(D-3) H a^{\prime}\right] i \Delta_{C}\left(x ; x^{\prime}\right) .
\end{aligned}
$$


Significant reductions are,

$$
\begin{aligned}
\partial_{0} \partial_{0}^{\prime} i \Delta_{A}= & \frac{i \delta^{D}\left(x-x^{\prime}\right)}{a^{D-2}}-\nabla^{2} i \Delta_{B} \\
\partial_{0} \partial_{0}^{\prime} i \Delta_{B}= & \frac{i \delta^{D}\left(x-x^{\prime}\right)}{a^{D-2}}-\nabla^{2} i \Delta_{A}+(D-2) H\left[a \partial_{0} i \Delta_{A}-a^{\prime} \partial_{0} i \Delta_{B}\right], \\
\partial_{0} \partial_{0}^{\prime} i \Delta_{C}= & \frac{i \delta^{D}\left(x-x^{\prime}\right)}{a^{D-2}}-\nabla^{2} i \Delta_{B}+(D-3) H\left[a \partial_{0} i \Delta_{B}-a^{\prime} \partial_{0} i \Delta_{C}\right] \\
& +(D-3) H^{2} a^{2} i \Delta_{B} .
\end{aligned}
$$

The reflection identities (85-86) can also be used to reach a manifestly reflection invariant form. Note also that we can ignore factors of $i \delta^{D}\left(x-x^{\prime}\right)$ because the coincidence limit of $i \Delta\left(x ; x^{\prime}\right)$ vanishes in dimensional regularization. The final result for this stage of the reduction is,

$$
\begin{gathered}
-i \widetilde{M}_{b b}^{2}\left(x ; x^{\prime}\right) \equiv-\frac{\kappa^{2}}{16}\left(\frac{D-2}{D-1}\right)^{2} i \Delta\left\{-2(D-2) H\left(a \partial_{0}^{\prime}+a^{\prime} \partial_{0}\right) \nabla^{2} i \Delta_{B}\right. \\
+2(D-2)(D-1) H^{2} a a^{\prime} \nabla^{2} i \Delta_{B}-2(D-1)^{2}(D-4) H^{3} a a^{\prime}\left(a \partial_{0}^{\prime}+a^{\prime} \partial_{0}\right) i \Delta_{C} \\
-\frac{2 D(D-1)^{2}(D-3)(D-4)}{(D-2)} H^{4} a^{2} a^{\prime 2} i \Delta_{C}+\frac{2(D-1)(D-2)}{(D-3)} H\left(a \partial_{0}+a^{\prime} \partial_{0}^{\prime}\right) \\
\times \nabla^{2}\left(i \Delta_{A}-i \Delta_{C}\right)-4(D-1) H^{2} a a^{\prime}\left(2+H^{2} a a^{\prime} \Delta x_{0}^{2}\right) \nabla^{2} i \Delta_{C} \\
-2\left(\frac{D-2}{D-3}\right) \nabla^{4}\left(i \Delta_{A}-i \Delta_{C}\right) .
\end{gathered}
$$

The next step is to act the derivatives using the identities,

$$
\begin{aligned}
&\left(a \partial_{0}^{\prime}+a^{\prime} \partial_{0}\right)\left[\frac{1}{\left(a a^{\prime} \Delta x^{2}\right)^{N}}\right]=-\frac{2 N H\|\Delta \vec{x}\|^{2}}{\left(a a^{\prime}\right)^{N-1} \Delta x^{2 N+2}}, \\
& \nabla^{2}\left[\frac{1}{\Delta x^{2 N}}\right]=-\frac{2 N(D-1)}{\Delta x^{2 N+2}}+\frac{4 N(N+1)\|\Delta \vec{x}\|^{2}}{\Delta x^{2 N+4}}, \\
& \nabla^{2}\left[\frac{\|\Delta \vec{x}\|^{2}}{\Delta x^{2 N+2}}\right]=\frac{2(D-1)}{\Delta x^{2 N+2}}-\frac{2(N+1)(D+3)\|\Delta \vec{x}\|^{2}}{\Delta x^{2 N+4}} \\
&+\frac{4(N+1)(N+2)\|\Delta \vec{x}\|^{4}}{\Delta x^{2 N+6}} .
\end{aligned}
$$

The final step is to extract derivatives using expressions $(\mathbf{7 2}-76)$ and the new relations,

$$
\frac{\|\Delta \vec{x}\|^{2}}{\Delta x^{2 D}} \longrightarrow\left[\frac{\nabla^{2}}{4(D-2)(D-1)}+\frac{\partial^{2}}{4(D-2)^{2}}\right] K i \delta^{D}\left(x-x^{\prime}\right)
$$




$$
\begin{aligned}
& -\left[\frac{\nabla^{2} \partial^{2}}{96}+\frac{\partial^{4}}{64}\right]\left[\frac{\ln \left(\mu^{2} \Delta x^{2}\right)}{\Delta x^{2}}\right], \\
& \frac{\|\Delta \vec{x}\|^{4}}{\Delta x^{2 D+2}} \longrightarrow\left[\frac{(D+1) \nabla^{2}}{4(D-2)(D-1) D}+\frac{(D+1) \partial^{2}}{8(D-2)^{2} D}\right] K i \delta^{D}\left(x-x^{\prime}\right) \\
& -\left[\frac{5 \nabla^{2} \partial^{2}}{384}+\frac{5 \partial^{4}}{512}\right]\left[\frac{\ln \left(\mu^{2} \Delta x^{2}\right)}{\Delta x^{2}}\right]+\frac{\nabla^{4}}{384}\left[\frac{1}{\Delta x^{2}}\right], \\
& \frac{\|\Delta \vec{x}\|^{2}}{\Delta x^{2 D-2}} \longrightarrow \frac{1}{2}\left(\frac{D-1}{D-2}\right) K i \delta^{D}\left(x-x^{\prime}\right)-\frac{3 \partial^{2}}{16}\left[\frac{\ln \left(\mu^{2} \Delta x^{2}\right)}{\Delta x^{2}}\right]+\frac{\nabla^{2}}{8}\left[\frac{1}{\Delta x^{2}}\right] \text {, } \\
& \frac{\|\Delta \vec{x}\|^{4}}{\Delta x^{2 D}} \longrightarrow \frac{1}{4}\left(\frac{D+1}{D-2}\right) K i \delta^{D}\left(x-x^{\prime}\right) \\
& -\frac{5 \partial^{2}}{32}\left[\frac{\ln \left(\mu^{2} \Delta x^{2}\right)}{\Delta x^{2}}\right]+\frac{5 \nabla^{2}}{24}\left[\frac{1}{\Delta x^{2}}\right]-\frac{\nabla^{4}}{96}\left[\ln \left(\mu^{2} \Delta x^{2}\right)\right],
\end{aligned}
$$

where we recall the definition (77) of the constant $K$. Amazingly, the final result turns out to be both finite and local,

$$
-i \widetilde{M}_{b b}^{2}\left(x ; x^{\prime}\right)=\frac{\kappa^{2} H^{2}}{32 \pi^{2}}\left\{\frac{1}{3} \partial^{2}-\frac{4}{9} \nabla^{2}+\frac{2}{3} H^{2} a^{2}\right\} i \delta^{4}\left(x-x^{\prime}\right) \text {. }
$$

\subsection{Total Primitive Contribution}

The total primitive contribution comes from combining expressions (41), (43), (46), (60), (81) and (98),

$$
\begin{gathered}
-i \widetilde{M}_{\text {prim }}^{2}\left(x ; x^{\prime}\right)=\frac{-\kappa^{2} A_{1} \partial^{2} \partial^{\prime 2}}{4(D-1)(D-3) H^{2}}\left[\frac{i \delta^{D}\left(x-x^{\prime}\right)}{\left(a a^{\prime}\right)^{\frac{D}{2}-1}}\right]-\frac{19 \kappa^{2} A_{1}}{12} \partial \cdot \partial^{\prime}\left[\frac{i \delta^{D}\left(x-x^{\prime}\right)}{\left(a a^{\prime}\right)^{\frac{D}{2}-2}}\right] \\
+\kappa^{2}\left(A_{0}+\frac{3}{2} A_{1}\right) \vec{\nabla} \cdot \vec{\nabla}^{\prime}\left[\frac{i \delta^{D}\left(x-x^{\prime}\right)}{\left(a a^{\prime}\right)^{\frac{D}{2}-2}}\right]+\frac{\kappa^{2} H^{2}}{12 \pi^{2}}\left[\frac{139}{24} \partial^{2}-\frac{17}{4} \nabla^{2}+\frac{1}{2} H^{2} a^{2}\right] i \delta^{4}\left(x-x^{\prime}\right) \\
+\frac{\kappa^{2} \partial^{2} \partial^{\prime 2}}{384 \pi^{4}}\left(\frac{1}{a a^{\prime}} \partial^{2}\left[\frac{\ln \left(\mu^{2} \Delta x^{2}\right)}{\Delta x^{2}}\right]\right)-\frac{\kappa^{2} H^{2}\left(19 \partial^{4}-18 \nabla^{2} \partial^{2}\right)}{384 \pi^{4}}\left[\frac{\ln \left(\mu^{2} \Delta x^{2}\right)}{\Delta x^{2}}\right] \\
+\frac{\kappa^{2} H^{2} \partial^{2} \nabla^{2}}{16 \pi^{4}}\left[\frac{\frac{1}{2} \ln \left(\frac{1}{4} H^{2} \Delta x^{2}\right)+1}{\Delta x^{2}}\right] .
\end{gathered}
$$

Recall that the constants $A_{0}$ and $A_{1}$ were defined in expression (61). Note also that we have expanded the complications functions of $D$ that multiply $A_{0}$ and $A_{1}$ around $D=4$ and used the limits,

$$
\lim _{D \rightarrow 4}(D-4) \times A_{0}=\lim _{D \rightarrow 4}(D-4) \times A_{1}=\frac{H^{2}}{4 \pi^{2}} .
$$


Finally, note that the flat space limit of our result comes entirely from the mixed contribution (81),

$$
\lim _{H \rightarrow 0}\left[-i \widetilde{M}_{\text {prim }}^{2}\left(x ; x^{\prime}\right)\right]=-\frac{\kappa^{2} \mu^{D-4} \Gamma\left(\frac{D}{2}\right) \partial^{4} i \delta^{D}\left(x-x^{\prime}\right)}{16 \pi^{\frac{D}{2}}(D-1)(D-3)(D-4)}+\frac{\kappa^{2} \partial^{6}}{384 \pi^{4}}\left[\frac{\ln \left(\mu^{2} \Delta x^{2}\right)}{\Delta x^{2}}\right] .
$$

It is cheering to see that the finite part of this obeys the flat space correspondence limit found by Fröb, cf. eqn (58) of [4].

\section{Renormalization}

On de Sitter background, and using our gauge [5, 6], four counterterms are required to renormalize $-i M^{2}\left(x ; x^{\prime}\right)$ at one loop order. Each involves two scalars and four derivatives distributed variously over the scalars and metrics. How to express them is motivated by the effects of conformal rescaling (4),

$$
\begin{aligned}
\sqrt{-g} R & =a^{D-2}\left[\sqrt{-\widetilde{g}} \widetilde{R}-2(D-1) H a \partial_{\mu}\left(\sqrt{-\widetilde{g}} \widetilde{g}^{\mu 0}\right)\right. \\
& \left.-D(D-1) H^{2} a^{2} \sqrt{-\widetilde{g}} \widetilde{g}^{00}\right], \\
\square \phi-\frac{1}{4}\left(\frac{D-2}{D-1}\right) R \phi= & \frac{1}{a^{\frac{D}{2}+1}}\left[\widetilde{\square} \widetilde{\phi}-\frac{1}{4}\left(\frac{D-2}{D-1}\right) \widetilde{R} \widetilde{\phi}\right]
\end{aligned}
$$

where the covariant scalar d'Alembertian is,

$$
\square \equiv \frac{1}{\sqrt{-g}} \partial_{\mu}\left(\sqrt{-g} g^{\mu \nu} \partial_{\nu}\right)
$$

The best arrangement of counterterms seems to be,

$$
\begin{aligned}
\Delta \mathcal{L}=-\frac{\alpha}{2}\left[\square \phi-\frac{1}{4}\left(\frac{D-2}{D-1}\right) R \phi\right]^{2} & \sqrt{-g}-\frac{\beta}{2}\left[\square \phi-\frac{1}{4}\left(\frac{D-2}{D-1}\right) R \phi\right] \frac{\phi R \sqrt{-g}}{D(D-1)} \\
& -\frac{\gamma}{2} \partial_{i} \phi \partial_{j} \phi g^{i j} \frac{R \sqrt{-g}}{D(D-1)}-\frac{\delta}{2} \frac{\phi^{2} R^{2} \sqrt{-g}}{D^{2}(D-1)^{2}} \cdot(105
\end{aligned}
$$

The noncovariant term proportional to $\gamma$ is the price of using a de Sitter

breaking gauge. Note also that a general metric background might require 
additional counterterms involving other curvatures which degenerate to the Ricci scalar on de Sitter, $R_{\mu \nu \rho \sigma}=\frac{1}{D(D-1)}\left(g_{\mu \rho} g_{\nu \sigma}-g_{\mu \sigma} g_{\nu \rho}\right) R$.

Specializing the counter-Lagrangian (105) to de Sitter gives,

$$
\left.\Delta \mathcal{L}\right|_{\text {de Sitter }}=-\frac{\alpha\left(\partial^{2} \widetilde{\phi}\right)^{2}}{2 a^{2}}-\frac{\beta}{2} \partial^{2} \widetilde{\phi} H^{2} \widetilde{\phi}-\frac{\gamma}{2} \partial_{i} \widetilde{\phi} \partial_{i} \widetilde{\phi} H^{2}-\frac{\delta}{2} \widetilde{\phi}^{2} H^{4} a^{2}
$$

The second variations of each of the four terms in the counter-action gives,

$$
\begin{aligned}
& \frac{i \delta^{2} \Delta S_{\alpha}}{\delta \widetilde{\phi}(x) \delta \widetilde{\phi}\left(x^{\prime}\right)}=-\alpha \partial^{2} \partial^{\prime 2}\left[\frac{i \delta^{D}\left(x-x^{\prime}\right)}{a a^{\prime}}\right], \\
& \frac{i \delta^{2} \Delta S_{\beta}}{\delta \widetilde{\phi}(x) \delta \widetilde{\phi}\left(x^{\prime}\right)}=-\beta H^{2} \partial^{2} i \delta^{D}\left(x-x^{\prime}\right), \\
& \frac{i \delta^{2} \Delta S_{\gamma}}{\delta \widetilde{\phi}(x) \delta \widetilde{\phi}\left(x^{\prime}\right)}=+\gamma H^{2} \nabla^{2} i \delta^{D}\left(x-x^{\prime}\right), \\
& \frac{i \delta^{2} \Delta S_{\delta}}{\delta \widetilde{\phi}(x) \delta \widetilde{\phi}\left(x^{\prime}\right)}=-\delta H^{4} a^{2} i \delta^{D}\left(x-x^{\prime}\right) .
\end{aligned}
$$

Comparison with the primitive result (99) implies the following values for the four counterterms,

$$
\begin{aligned}
\alpha & =\frac{-\kappa^{2} A_{1}}{4(D-1)(D-3) H^{2}}+\alpha_{\mathrm{fin}}, \\
\beta & =\frac{19 \kappa^{2} A_{1}}{12 H^{2}}+\frac{139 \kappa^{2}}{288 \pi^{2}}+\beta_{\mathrm{fin}}, \\
\gamma & =\frac{\kappa^{2}}{H^{2}}\left(A_{0}+\frac{3}{2} A_{1}\right)+\frac{17 \kappa^{2}}{48 \pi^{2}}+\gamma_{\mathrm{fin}}, \\
\delta & =0+\frac{\kappa^{2}}{24 \pi^{2}}+\delta_{\mathrm{fin}} .
\end{aligned}
$$

With these choices the renormalized result becomes,

$$
\begin{aligned}
& -i \widetilde{M}_{\mathrm{ren}}^{2}\left(x ; x^{\prime}\right)=\kappa^{2} \partial^{2} \partial^{\prime 2}\left\{\left[\frac{\ln \left(a a^{\prime}\right)}{96 \pi^{2}}-\alpha_{\mathrm{fin}}\right] \frac{i \delta^{4}\left(x-x^{\prime}\right)}{a a^{\prime}}\right\} \\
& +\kappa^{2} H^{2} \partial \cdot \partial^{\prime}\left\{\left[\frac{19 \ln \left(a a^{\prime}\right)}{96 \pi^{2}}+\beta_{\mathrm{fin}}\right] i \delta^{4}\left(x-x^{\prime}\right)\right\} \\
& -\kappa^{2} H^{2} \vec{\nabla} \cdot \vec{\nabla}^{\prime}\left\{\left[\frac{5 \ln \left(a a^{\prime}\right)}{16 \pi^{2}}+\gamma_{\mathrm{fin}}\right] i \delta^{4}\left(x-x^{\prime}\right)\right\}-\delta_{\mathrm{fin}} \kappa^{2} H^{4} a^{2} i \delta^{4}\left(x-x^{\prime}\right)
\end{aligned}
$$




$$
\begin{array}{r}
+\frac{\kappa^{2} \partial^{2} \partial^{\prime 2}}{384 \pi^{4}}\left(\frac{1}{a a^{\prime}} \partial^{2}\left[\frac{\ln \left(\mu^{2} \Delta x^{2}\right)}{\Delta x^{2}}\right]\right)- \\
-\frac{\kappa^{2} H^{2}\left(19 \partial^{4}-18 \nabla^{2} \partial^{2}\right)}{384 \pi^{4}}\left[\frac{\ln \left(\mu^{2} \Delta x^{2}\right)}{\Delta x^{2}}\right] \\
+\frac{\kappa^{2} H^{2} \partial^{2} \nabla^{2}}{16 \pi^{4}}\left[\frac{\frac{1}{2} \ln \left(\frac{1}{4} H^{2} \Delta x^{2}\right)+1}{\Delta x^{2}}\right]
\end{array}
$$

\section{Conclusions}

The point of this exercise has been to begin the process of purging gauge dependence from the linearized effective field equations in cosmology by including quantum gravitational corrections from the source which disturbs the effective field and from the observer who measures the disturbance [24]. To simplify the tensor algebra it makes sense to work with the effective field equations for a scalar. We might have employed the existing result for the self-mass of a massless, minimally coupled scalar [10]. However, that is known to cause no secular growth for the scalar mode function [17], and the classical solution for the exchange potential [28] is so complicated that computing its one loop correction would be daunting. The next simplest sort of scalar is the conformally coupled case; with an arbitrary $R \phi^{2}$ coupling the scalar propagator becomes much more complicated.

Our result for the one graviton loop correction to the self-mass of a conformally coupled scalar is equation (115). The linearized, effective field equation for this scalar is,

$$
\partial^{2} \widetilde{\phi}(x)-\int d^{4} x^{\prime} \widetilde{M}^{2}\left(x ; x^{\prime}\right) \widetilde{\phi}\left(x^{\prime}\right)=\widetilde{J}(x),
$$

where tildes denote conformal re-scaling (4) and we employ the SchwingerKeldysh formalism [46 53] to make the effective field equation both real and causal. This is a diagrammatic technique for computing true expectation values which is almost as simple as the Feynman rules that produce the sorts of in-out matrix elements we have computed in this paper. For our purposes the rules are [12]:

- Every line carries a \pm polarity corresponding to the usual case of a field being evolved forward in time $(+)$ or being evolved backwards $(-)$;

- The ++ propagator agrees with the Feynman propagator, and the -propagator is its complex conjugate, while the +- and -+ propagators 
are obtained by replacing the interval $\Delta x^{2}$ in expression (20) with,

$$
\begin{aligned}
& \Delta x_{+-}^{2} \equiv\left\|\vec{x}-\vec{x}^{\prime}\right\|^{2}-\left(\eta-\eta^{\prime}+i \epsilon\right)^{2}, \\
& \Delta x_{-+}^{2} \equiv\left\|\vec{x}-\vec{x}^{\prime}\right\|^{2}-\left(\eta-\eta^{\prime}-i \epsilon\right)^{2} ;
\end{aligned}
$$

- There are only all + vertices, which are the same as for the Feynman rules, and - vertices, which are complex conjugated;

- Every 1PI $N$-point function of the Feynman rules corresponds to $2^{N}$ $1 \mathrm{PI} N$-point functions in the Schwinger-Keldysh formalism; and

- The effective field equation (116) uses,

$$
\widetilde{M}^{2}\left(x ; x^{\prime}\right)=\widetilde{M}_{++}^{2}\left(x ; x^{\prime}\right)+\widetilde{M}_{+-}^{2}\left(x ; x^{\prime}\right) .
$$

It is therefore trivial to convert our in-out result (115) into the analogous Schwinger-Keldysh result.

The next step in our program is to solve equation (116) for one loop corrections to the plane wave mode function and to the exchange potential:

$$
\begin{aligned}
\widetilde{J}(\eta, \vec{x})=0 & \Longrightarrow \widetilde{\phi}(\eta, \vec{x})=\left\{e^{-i k \eta}+\kappa^{2} u_{1}(\eta, k)+O\left(\kappa^{4}\right)\right\} e^{i \vec{k} \cdot \vec{x}}, \\
\widetilde{J}(\eta, \vec{x})=\delta^{3}(\vec{x}) & \Longrightarrow-\frac{1}{4 \pi\|\vec{x}\|}\left\{1+\kappa^{2} \Phi_{1}(\eta,\|\vec{x}\|)+O\left(\kappa^{4}\right)\right\} .
\end{aligned}
$$

Although we do not need the $\delta$ counterterm (110) to remove ultraviolet divergences, we expect that its finite part in expression (114) can be chosen to free $u_{1}(\eta, k)$ of any secular enhancement. However, we also anticipate that the one loop correction to the potential will take the same form that was found for scalar corrections to gravitational potentials [54],

$$
\Phi_{1}(\eta, \vec{x})=\frac{k_{1}}{a^{2}\|\vec{x}\|^{2}}+k_{2} H^{2} \ln (a)+k_{3} H^{2} \ln (a H\|\vec{x}\|),
$$

where $k_{1}, k_{2}$ and $k_{3}$ are constants. The term proportional to $k_{1}$ descends from known effects in flat space and is anyway negligible at large distances. However, the potentially large logarithms proportional to $k_{2}$ and $k_{3}$ are de Sitter effects associated with inflationary particle production. It is the gauge dependence of these effects that we seek to establish by checking that they persist when source and observer effects have been included. The next steps in our program are therefore: 
1. Solve equation (116) for the case (121) to verify (122); and

2. Show that the constants $k_{2}$ and/or $k_{3}$ are nonzero when source and observer corrections have been included.

It is worth mentioning other approaches to defining gauge independent correlators. The simplest is by taking the expectation values of (necessarily nonlocal) invariant operators [4, 21, 55, 60]. A closely related program is the gravitational dressing advocated by Giddings and collaborators [61 64]. Proposals have also been made for defining invariant observables in loop quantum gravity [65, 66] and in algebraic quantum field theory [67]. Finally, we should mention the technique of cosmological averaging [68].

Before closing we should also comment on the previous computation of $-i \widetilde{M}^{2}\left(x ; x^{\prime}\right)$ by Boran, Kahya and Park [1,2]. Although this was a significant piece of work, it suffers from three problems. First, the earlier result was expressed in terms of unwieldy, de Sitter covariant differential operators, rather than the simple, de Sitter breaking operators $\partial^{2}$ and $\nabla^{2}$ that we employed in expression (115). The de Sitter operators are so complicated that comparison is not easy but the two results do not agree. Second, Fröb has identified a problem in the flat space correspondence limit of the earlier computation [4], which our result avoids. Finally, using the earlier result to solve equation (116) for case (120) results in one loop corrections $u_{1}(\eta, k)$ that grow like $\ln (a)$ [3], whereas it is obvious from our result (115) that the arbitrary constant $\delta_{\text {fin }}$ can be chosen to absorb any such enhancement.

\section{Acknowledgements}

This work was partially supported by the Fonds de la Recherche Scientifique - FNRS under Grant IISN 4.4517.08 - Theory of fundamental interactions; by Taiwan MOST grant 108-2112-M-006-004; by the D-ITP consortium, a program of the Netherlands Organization for Scientific Research (NWO) that is funded by the Dutch Ministry of Education, Culture and Science (OCW); by NSF grants PHY-1806218 and PHY-1912484; and by the Institute for Fundamental Theory at the University of Florida. The authors also wish to express their gratitude to NCKU for providing office space during this project. 


\section{References}

[1] S. Boran, E. O. Kahya and S. Park, Phys. Rev. D 90, no. 12, 124054 (2014) doi:10.1103/PhysRevD.90.124054 [arXiv:1409.7753 [gr-qc]].

[2] S. Boran, E. O. Kahya and S. Park, Phys. Rev. D 96, no. 2, 025001 (2017) doi:10.1103/PhysRevD.96.025001 [arXiv:1704.05880 [gr-qc]].

[3] S. Boran, E. O. Kahya and S. Park, Phys. Rev. D 96, no. 10, 105003 (2017) Erratum: [Phys. Rev. D 98, no. 2, 029903 (2018)] doi:10.1103/PhysRevD.98.029903, 10.1103/PhysRevD.96.105003 arXiv:1708.01831 [gr-qc]].

[4] M. B. Fröb, Class. Quant. Grav. 35, no. 3, 035005 (2018) doi:10.1088/1361-6382/aa9ad1 [arXiv:1706.01891 [hep-th]].

[5] N. C. Tsamis and R. P. Woodard, Commun. Math. Phys. 162, 217 (1994). doi:10.1007/BF02102015

[6] R. P. Woodard, gr-qc/0408002.

[7] N. C. Tsamis and R. P. Woodard, Phys. Rev. D 54, 2621 (1996) doi:10.1103/PhysRevD.54.2621 [hep-ph/9602317].

[8] S. P. Miao and R. P. Woodard, Class. Quant. Grav. 23, 1721 (2006) doi:10.1088/0264-9381/23/5/016 |gr-qc/0511140.

[9] S. P. Miao, Phys. Rev. D 86, $104051 \quad$ (2012) doi:10.1103/PhysRevD.86.104051 [arXiv:1207.5241 [gr-qc]].

[10] E. O. Kahya and R. P. Woodard, Phys. Rev. D 76, 124005 (2007) doi:10.1103/PhysRevD.76.124005 arXiv:0709.0536 [gr-qc]].

[11] K. E. Leonard and R. P. Woodard, Class. Quant. Grav. 31, 015010 (2014) doi:10.1088/0264-9381/31/1/015010 [arXiv:1304.7265 [gr-qc]].

[12] L. H. Ford and R. P. Woodard, Class. Quant. Grav. 22, 1637 (2005) doi:10.1088/0264-9381/22/9/011 |gr-qc/0411003|.

[13] T. Prokopec, O. Tornkvist and R. P. Woodard, Phys. Rev. Lett. 89, 101301 (2002) doi:10.1103/PhysRevLett.89.101301 [astro-ph/0205331. 
[14] T. Prokopec and R. P. Woodard, Am. J. Phys. 72, 60 (2004) doi:10.1119/1.1596180 astro-ph/0303358.

[15] P. J. Mora, N. C. Tsamis and R. P. Woodard, JCAP 1310, 018 (2013) doi:10.1088/1475-7516/2013/10/018 arXiv:1307.1422 [gr-qc]].

[16] S. P. Miao and R. P. Woodard, Phys. Rev. D 74, 024021 (2006) doi:10.1103/PhysRevD.74.024021 [gr-qc/0603135].

[17] E. O. Kahya and R. P. Woodard, Phys. Rev. D 77, 084012 (2008) doi:10.1103/PhysRevD.77.084012 arXiv:0710.5282 [gr-qc]].

[18] C. L. Wang and R. P. Woodard, Phys. Rev. D 91, no. 12, 124054 (2015) doi:10.1103/PhysRevD.91.124054 arXiv:1408.1448 [gr-qc]].

[19] D. Glavan, S. P. Miao, T. Prokopec and R. P. Woodard, Class. Quant. Grav. 31, 175002 (2014) doi:10.1088/0264-9381/31/17/175002 arXiv:1308.3453 [gr-qc]].

[20] K. E. Leonard and R. P. Woodard, Phys. Rev. D 85, 104048 (2012) doi:10.1103/PhysRevD.85.104048 arXiv:1202.5800 [gr-qc]].

[21] S. P. Miao and R. P. Woodard, JCAP 1207, 008 (2012) doi:10.1088/1475-7516/2012/07/008 arXiv:1204.1784 [astro-ph.CO]].

[22] D. Glavan, S. P. Miao, T. Prokopec and R. P. Woodard, Class. Quant. Grav. 32, no. 19, 195014 (2015) doi:10.1088/0264-9381/32/19/195014 arXiv:1504.00894 [gr-qc]].

[23] D. Glavan, S. P. Miao, T. Prokopec and R. P. Woodard, Class. Quant. Grav. 34, no. 8, 085002 (2017) doi:10.1088/1361-6382/aa61da arXiv:1609.00386 [gr-qc]].

[24] S. P. Miao, T. Prokopec and R. P. Woodard, Phys. Rev. D 96, no. 10, 104029 (2017) doi:10.1103/PhysRevD.96.104029 arXiv:1708.06239 [gr-qc]].

[25] J. F. Donoghue, Phys. Rev. D 50, 3874 (1994) doi:10.1103/PhysRevD.50.3874 [gr-qc/9405057].

[26] J. F. Donoghue and T. Torma, Phys. Rev. D 54, 4963 (1996) doi:10.1103/PhysRevD.54.4963 [hep-th/9602121]. 
[27] E. Lifshitz, J. Phys. (USSR) 10, no. 2, 116 (1946) [Gen. Rel. Grav. 49, no. 2, 18 (2017)]. doi:10.1016/B978-0-08-036364-6.50020-X, $10.1007 / \mathrm{s} 10714-016-2165-8$

[28] D. Glavan, S. P. Miao, T. Prokopec and R. P. Woodard, Phys. Lett. B 798, 134944 (2019) doi:10.1016/j.physletb.2019.134944 arXiv:1908.11113 [gr-qc]].

[29] S. P. Miao and R. P. Woodard, Class. Quant. Grav. 25, 145009 (2008) doi:10.1088/0264-9381/25/14/145009 [arXiv:0803.2377 [gr-qc]].

[30] A. Higuchi and S. S. Kouris, Class. Quant. Grav. 18, 4317 (2001) doi:10.1088/0264-9381/18/20/311 [gr-qc/0107036].

[31] S. P. Miao, N. C. Tsamis and R. P. Woodard, J. Math. Phys. 50, 122502 (2009) doi:10.1063/1.3266179 arXiv:0907.4930 [gr-qc]].

[32] S. P. Miao, N. C. Tsamis and R. P. Woodard, J. Math. Phys. 52, 122301 (2011) doi:10.1063/1.3664760 [arXiv:1106.0925 [gr-qc]].

[33] M. Faizal and A. Higuchi, Phys. Rev. D 85, 124021 (2012) doi:10.1103/PhysRevD.85.124021 [arXiv:1107.0395 [gr-qc]].

[34] A. Higuchi, D. Marolf and I. A. Morrison, Class. Quant. Grav. 28, 245012 (2011) doi:10.1088/0264-9381/28/24/245012 arXiv:1107.2712 [hep-th]].

[35] S. P. Miao, N. C. Tsamis and R. P. Woodard, Class. Quant. Grav. 28, 245013 (2011) doi:10.1088/0264-9381/28/24/245013 arXiv:1107.4733 [gr-qc]].

[36] I. A. Morrison, arXiv:1302.1860 [gr-qc].

[37] S. P. Miao, P. J. Mora, N. C. Tsamis and R. P. Woodard, Phys. Rev. D 89, no. 10, 104004 (2014) doi:10.1103/PhysRevD.89.104004 arXiv:1306.5410 [gr-qc]].

[38] M. B. Fröb, JCAP 1412, 010 (2014) doi:10.1088/14757516/2014/12/010 [arXiv:1409.7964 [hep-th]].

[39] R. P. Woodard, JHEP 1605, 152 (2016) doi:10.1007/JHEP05(2016)152 arXiv:1506.04252 [gr-qc]]. 
[40] V. K. Onemli and R. P. Woodard, Phys. Rev. D 70, 107301 (2004) doi:10.1103/PhysRevD.70.107301 [gr-qc/0406098].

[41] K. Peeters, hep-th/0701238.

[42] K. Peeters, Journal of Open Source Software 3 (2018) no.32, 1118. doi: $10.21105 /$ joss. 01118

[43] V. K. Onemli and R. P. Woodard, Class. Quant. Grav. 19, 4607 (2002) doi:10.1088/0264-9381/19/17/311 [gr-qc/0204065].

[44] N. C. Tsamis and R. P. Woodard, Phys. Lett. B 292, 269 (1992). doi:10.1016/0370-2693(92)91174-8

[45] S. P. Miao, N. C. Tsamis and R. P. Woodard, J. Math. Phys. 51, 072503 (2010) doi:10.1063/1.3448926 [arXiv:1002.4037 [gr-qc]].

[46] J. S. Schwinger, J. Math. Phys. 2, 407 (1961). doi:10.1063/1.1703727

[47] K. T. Mahanthappa, Phys. Rev. 126, 329 (1962). doi:10.1103/PhysRev.126.329

[48] P. M. Bakshi and K. T. Mahanthappa, J. Math. Phys. 4, 1 (1963). doi:10.1063/1.1703883

[49] P. M. Bakshi and K. T. Mahanthappa, J. Math. Phys. 4, 12 (1963). doi:10.1063/1.1703879

[50] L. V. Keldysh, Zh. Eksp. Teor. Fiz. 47, 1515 (1964) [Sov. Phys. JETP 20, 1018 (1965)].

[51] K. c. Chou, Z. b. Su, B. l. Hao and L. Yu, Phys. Rept. 118, 1 (1985). doi:10.1016/0370-1573(85)90136-X

[52] R. D. Jordan, Phys. Rev. D 33, 444 (1986). doi:10.1103/PhysRevD.33.444

[53] E. Calzetta and B. L. Hu, Phys. Rev. D 35, 495 (1987). doi:10.1103/PhysRevD.35.495

[54] S. Park, T. Prokopec and R. P. Woodard, JHEP 1601, 074 (2016) doi:10.1007/JHEP01(2016)074 [arXiv:1510.03352 [gr-qc]]. 
[55] N. C. Tsamis and R. P. Woodard, Annals Phys. 215, 96 (1992). doi:10.1016/0003-4916(92)90301-2

[56] S. P. Miao, N. C. Tsamis and R. P. Woodard, Phys. Rev. D 95, no. 12, 125008 (2017) doi:10.1103/PhysRevD.95.125008 [arXiv:1702.05694 [gr-qc]].

[57] M. B. Fröb, Class. Quant. Grav. 35, no. 5, 055006 (2018) doi:10.1088/1361-6382/aaa74c arXiv:1710.00839 [gr-qc]].

[58] M. B. Fröb and W. C. C. Lima, Class. Quant. Grav. 35, no. 9, 095010 (2018) doi:10.1088/1361-6382/aab427 [arXiv:1711.08470 [gr-qc]].

[59] M. B. Fröb, Class. Quant. Grav. 36, no. 9, 095010 (2019) doi:10.1088/1361-6382/ab10fb arXiv:1806.11124 [gr-qc]].

[60] L. Chataignier, Phys. Rev. D 101, no. 8, 086001 (2020) doi:10.1103/PhysRevD.101.086001 [arXiv:1910.02998 [gr-qc]].

[61] W. Donnelly and S. B. Giddings, Phys. Rev. D 94, no. 10, 104038 (2016) doi:10.1103/PhysRevD.94.104038 arXiv:1607.01025 [hep-th]].

[62] S. B. Giddings and A. Kinsella, JHEP 1811, 074 (2018) doi:10.1007/JHEP11(2018)074 [arXiv:1802.01602 [hep-th]].

[63] S. B. Giddings, Phys. Rev. D 100, no. 12, 126001 (2019) doi:10.1103/PhysRevD.100.126001 [arXiv:1903.06160 [hep-th]].

[64] S. Giddings and S. Weinberg, arXiv:1911.09115 [hep-th].

[65] K. Giesel and A. Herzog, Int. J. Mod. Phys. D 27, no. 08, 1830005 (2018) doi:10.1142/S0218271818300057 [arXiv:1712.09878 [gr-qc]].

[66] K. Giesel, A. Herzog and P. Singh, Class. Quant. Grav. 35, no. 15, 155012 (2018) doi:10.1088/1361-6382/aacda2 [arXiv:1801.09630 [gr-qc]].

[67] R. Brunetti, K. Fredenhagen, T. P. Hack, N. Pinamonti and K. Rejzner, JHEP 1608, 032 (2016) doi:10.1007/JHEP08(2016)032 arXiv:1605.02573 [gr-qc]].

[68] G. Fanizza, M. Gasperini, G. Marozzi and G. Veneziano, JCAP 2002, 017 (2020) doi:10.1088/1475-7516/2020/02/017 arXiv:1911.09469 [grqc]]. 